\title{
Energy Decompositions for Moist Boussinesq and Anelastic Equations with Phase Changes $\mathscr{0}$
}

\author{
DAVID H. MARSICO \\ Department of Mathematics, University of Wisconsin-Madison, Madison, Wisconsin \\ LESLIE M. SMITH \\ Department of Mathematics, and Department of Engineering Physics, University of Wisconsin-Madison, Madison, Wisconsin \\ SAMUEL N. STECHMANN \\ Department of Mathematics, and Department of Atmospheric and Oceanic Sciences, \\ University of Wisconsin-Madison, Madison, Wisconsin
}

(Manuscript received 29 March 2019, in final form 24 July 2019)

\begin{abstract}
To define a conserved energy for an atmosphere with phase changes of water (such as vapor and liquid), motivation in the past has come from generalizations of dry energies-in particular, from gravitational potential energy $\rho g z$. Here a new definition of moist energy is introduced, and it generalizes another form of dry potential energy, proportional to $\theta^{2}$, which is valuable since it is manifestly quadratic and positive definite. The moist potential energy here is piecewise quadratic and can be decomposed into three parts, proportional to $b_{u}^{2} H_{u}, b_{s}^{2} H_{s}$, and $M^{2} H_{u}$, which represent, respectively, buoyant energies and a moist latent energy that is released upon a change of phase. The Heaviside functions $H_{u}$ and $H_{s}$ indicate the unsaturated and saturated phases, respectively. The $M^{2}$ energy is also associated with an additional eigenmode that arises for a moist atmosphere but not a dry atmosphere. Both the Boussinesq and anelastic equations are examined, and similar energy decompositions are shown in both cases, although the anelastic energy is not quadratic. Extensions that include cloud microphysics are also discussed, such as the Kessler warm-rain scheme. As an application, empirical orthogonal function (EOF) analysis is considered, using a piecewise quadratic moist energy as a weighted energy in contrast to the standard $L^{2}$ energy. By incorporating information about phase changes into the energy, the leading EOF modes become fundamentally different and capture the variability of the cloud layer rather than the dry subcloud layer.
\end{abstract}

\section{Introduction}

The main topic of this paper is conserved energies for atmospheric dynamics, in the case including phase changes of water-for example, vapor and liquid phases. As motivation, we will briefly discuss energies for dry dynamics (without water of any phase), followed by motivating discussion of energies for moist dynamics.

Two energies can be shown to be conserved for a $d r y$ Boussinesq atmosphere. The two energy densities are

Supplemental information related to this paper is available at the Journals Online website: https://doi.org/10.1175/JAS-D-190080.s1.

Corresponding author: David Marsico, marsico@wisc.edu

$$
E_{1}=\frac{1}{2}|\mathbf{u}|^{2}+\frac{b^{2}}{2 N^{2}}
$$

and

$$
\tilde{E}_{2}=\frac{1}{2}|\mathbf{u}|^{2}-b z
$$

or the related quantity

$$
E_{2}=\frac{1}{2}|\mathbf{u}|^{2}-\int_{a}^{z}\left(b^{\text {tot }}-N^{2} z^{\prime}\right) d z^{\prime} .
$$

An anelastic atmosphere also has a conserved energy, and is also considered in the present paper, but we restrict attention to the Boussinesq case for the moment for motivational purposes. For references, 
see Young (2010), Vallis (2006, chapter 2), or Ingersoll (2005), and earlier related work of Holliday and McIntyre (1981) and Andrews (1981). Here, $\mathbf{u}$ is the velocity, $b$ is the buoyancy, $N$ is the constant buoyancy frequency, $b^{\text {tot }}=b+N^{2} z, a$ is an arbitrary reference height, and the integration in (2) is done while keeping $b^{\text {tot }}$ constant. Each of the energies has its advantageous properties: for example, $E_{2}$ and $\tilde{E}_{2}$ use potential energies that are natural generalizations of the gravitational potential energy $\rho g z$, and $E_{1}$ has the property of being quadratic and clearly positive definite.

For a moist atmosphere with phase changes, on the other hand, a generalization of the potential energy in (2) has been proposed as

$$
E_{2}=\frac{1}{2}|\mathbf{u}|^{2}-\int_{a}^{z} g b\left(\theta_{e}^{\text {tot }}, q_{t}^{\text {tot }}, z^{\prime}\right) d z^{\prime}
$$

For references, see Pauluis (2008), Young (2010), Hernandez-Duenas et al. (2013, 2015), and Tailleux (2013). Here, the buoyancy $b$ is a function of the equivalent potential temperature $\theta_{e}^{\text {tot }}$, the total water mixing ratio $q_{t}^{\text {tot }}$, and height $z$, and again the integral is taken with $\theta_{e}^{\text {tot }}$ and $q_{t}^{\text {tot }}$ held fixed.

The main purpose of the present paper is to show that the dry quadratic energy in (1) can also be generalized for a moist atmosphere with phase changes. Specifically, in this paper, we introduce the piecewise quadratic conserved energy

$$
\begin{aligned}
E_{1}= & \frac{1}{2}|\mathbf{u}|^{2}+\frac{1}{2}\left(\frac{b_{u}^{2}}{N_{u}^{2}}\right) H_{u}+\frac{1}{2}\left(\frac{b_{s}^{2}}{N_{s}^{2}}\right) H_{s} \\
& +\frac{1}{2}\left(\frac{N_{u}^{2} N_{s}^{2}}{N_{u}^{2}-N_{s}^{2}}\right) M^{2} H_{u} .
\end{aligned}
$$

Here, $M=N_{u}^{-2} b_{u}-N_{s}^{-2} b_{s}, H_{u}$ is a Heaviside function indicating unsaturated regions, and $H_{s}=1-H_{u}$ is a Heaviside function indicating saturated regions. The search for an energy of this form was motivated by a similar energy for quasigeostrophic equations with precipitation and phase changes (Smith and Stechmann 2017). The energy in (4) has several advantageous properties: it is piecewise quadratic, manifestly positive definite, and the potential energy has been decomposed into buoyant contributions from each phase (proportional to $b_{u}^{2} H_{u}$ and $b_{s}^{2} H_{s}$ ) and a latent moist energy (proportional to $M^{2} H_{u}$ ). Furthermore, the piecewise quadratic energy in (4) is equivalent to the earlier energy in (3) aside from the addition of a material invariant (a function of the conserved variables $\theta_{e}^{\text {tot }}$ and $\left.q_{t}^{\text {tot }}\right)$.

Another moist energy that has been considered in the past is the moist available potential energy (APE) of
Lorenz (1955, 1978; see also Pauluis 2007, and references therein). It can be viewed as a special case of the potential energy, where a specific background state has been selected (e.g., Tailleux 2013; Stansifer et al. 2017). In the present paper, a more general setup is considered where the reference state is not constrained in the sense of Lorenz's APE but could be chosen in that way if desired. Also, Lorenz's APE is often considered along with the assumption of hydrostatic balance, whereas the present paper considers nonhydrostatic motions according to the Boussinesq or anelastic equations.

Yet another type of moist energy that is often considered is the moist static energy (e.g., Emanuel 1994). It is a thermodynamic quantity, not including a contribution from kinetic energy, and it is conserved under the assumption of hydrostatic balance (whereas, as mentioned above, the present paper considers nonhydrostatic motions according to the Boussinesq or anelastic equations).

The remainder of the paper is organized as follows. In section 2 a we describe in detail the dry Boussinesq equations, as well as discuss $E_{1}$ and $E_{2}$ in slightly more depth. Thus, section $2 b$ contains a description of the moist, nonprecipitating Boussinesq equations with phase changes. In section $2 \mathrm{c}$ we show that it is still possible to obtain a quadratic, positive-definite energy even in the presence of phase changes. While the Boussinesq case provides a natural starting point and simple analytical expressions, the real atmosphere has nonconstant buoyancy frequency and other complications. As steps to moving beyond this simple case, subsequent sections will incorporate increasing amounts of thermodynamic and microphysical complexity. In section 3, we consider the anelastic equations, and while we cannot formulate a quadratic energy, we can still decompose a total energy into buoyant energy and latent energy released at the interface between different phases. In section 4, we consider a Kessler warm-rain parameterization of cloud microphysics, and the Fast Autoconversion and Rain Evaporation (FARE) model of Hernandez-Duenas et al. (2013). In the former case, while we cannot obtain a conserved energy, we can still derive an energy principle that involves the source terms representing cloud microphysical processes. In the latter case, we show that there exists a unique quadratic, positive-definite energy, which is conserved in the absence of rainfall. In section 5 , we discuss an application to empirical orthogonal function analysis, and we show that taking phase changes into consideration results in significant differences of the EOF modes. We conclude with a discussion in section 6 . 


\section{Boussinesq equations}

\section{a. Energetics for the dry Boussinesq equations}

We will begin by describing energetics for a dry Boussinesq system with no phase changes. For derivations of the Boussinesq equations, see, for example, Vallis (2006). The equations for a dry atmosphere with no phase changes are

$$
\begin{aligned}
\frac{D \mathbf{u}}{D t} & =-\nabla \phi+b \hat{\mathbf{z}}, \\
\frac{D b}{D t}+N^{2} w & =0, \\
\nabla \cdot \mathbf{u} & =0,
\end{aligned}
$$

where $\mathbf{u}(\mathbf{x}, t)$ is the velocity vector, $\phi=p^{\prime} / \rho_{0}, p^{\prime}$ is the pressure, $\rho_{0}$ is a constant background density, $b$ is the buoyancy, and $N^{2}$ is the squared buoyancy frequency and is taken to be a constant. The buoyancy is $b=g \theta^{\prime} / \theta_{0}$, where $\theta^{\prime}$ is the potential temperature, and we have assumed that the total thermodynamic variables of potential temperature $\theta(\mathbf{x}, t)$ and pressure $p(\mathbf{x}, t)$ have been decomposed into background and fluctuating parts; $\theta(\mathbf{x}, t)=$ $\tilde{\theta}(z)+\theta^{\prime}(\mathbf{x}, t)$ and $p(\mathbf{x}, t)=\tilde{p}(z)+p^{\prime}(\mathbf{x}, t)$, where the background pressure $\tilde{p}(z)$ is hydrostatically balanced.

The total energy of a system governed by $(5 a)-(5 c)$ can be decomposed into both kinetic and potential energy parts. There are, however, different ways of specifying the potential energy component, which give rise to the two energies, $E_{1}$ and $E_{2}$, given by (1) and (2). These two energies satisfy the equations

$$
\frac{\partial E}{\partial t}+\nabla \cdot[\mathbf{u}(E+\phi)]=0,
$$

where $E$ can be $E_{1}$ or $E_{2}$. While there are clear differences between these two energies, they are still related. It can be shown that

$$
E_{2}=E_{1}-\frac{1}{2 N^{2}}\left[b+N^{2}(z-a)\right]^{2} .
$$

From (5b), we see that $b+N^{2}(z-a)$ is a conserved variable, which implies that $E_{1}$ and $E_{2}$ differ by a quadratic material invariant. In subsequent sections, we will define total energies analogous to those given in (1) and (2) that include the effects of moisture and phase changes, and show that these energies satisfy an equation similar to (7).

\section{b. Moist Boussinesq equations with phase changes}

Now we incorporate moisture and phase changes into the Boussinesq equations. That is, we assume that moisture is present and can be in the form of either water vapor or liquid water. Regions where liquid water is present are referred to as saturated regions, and regions without liquid water are referred to as unsaturated regions. If we assume that liquid water does not precipitate, then the equations are

$$
\begin{aligned}
\frac{D \mathbf{u}}{D t} & =-\nabla \phi+\left(b_{u} H_{u}+b_{s} H_{s}\right) \hat{\mathbf{z}}, \\
\frac{D b_{u}}{D t}+N_{u}^{2} w & =0, \\
\frac{D b_{s}}{D t}+N_{s}^{2} w & =0, \\
\nabla \cdot \mathbf{u} & =0,
\end{aligned}
$$

where $\mathbf{u}$ is the velocity vector, and $b_{u}$ is the unsaturated buoyancy, and $b_{s}$ is the saturated buoyancy. The variables $b_{u}$ and $b_{s}$ are defined over the whole domain and can be expressed in terms of equivalent potential temperature $\theta_{e}$, total water $q_{t}$, and prescribed saturation mixing ratio $q_{v s}$ :

$$
\begin{aligned}
& b_{u}=g\left[\frac{\theta_{e}}{\theta_{0}}+\left(R_{v d}-\frac{L_{v}}{c_{p} \theta_{0}}\right) q_{t}\right], \\
& b_{s}=g\left[\frac{\theta_{e}}{\theta_{0}}+\left(R_{v d}-\frac{L_{v}}{c_{p} \theta_{0}}+1\right) q_{v s}(z)-q_{t}\right] .
\end{aligned}
$$

The Heaviside functions $H_{u}$ and $H_{s}$ indicate the unsaturated and saturated phases, respectively, and are defined in terms of $b_{u}$ and $b_{s}$ :

$$
H_{u}=\left\{\begin{array}{lll}
1 & \text { if } \quad b_{u}>b_{s} \\
0 & \text { if } \quad b_{u} \leq b_{s}
\end{array}\right.
$$

and $H_{s}=1-H_{u}$. More precisely, $H_{u}(\mathbf{x}, t)$ is a function of $\mathbf{x}$ and $t$ and is defined via function composition as $H_{u}(\mathbf{x}, t)=H\left(b_{u}-b_{s}\right)$, where $b_{u}$ and $b_{s}$ are themselves functions of $\mathbf{x}$ and $t$, and where $H(s)$ is the Heaviside function, which takes the value of 1 for $s>0$ and 0 for $s \leq 0$. The constants $N_{u}$ and $N_{s}$ are the buoyancy frequencies for the unsaturated and saturated phases, respectively, and are defined as

$$
\begin{aligned}
& N_{u}^{2}=g \frac{d}{d z}\left[\frac{\tilde{\theta}_{e}}{\theta_{0}}+\left(R_{v d}-\frac{L_{v}}{c_{p} \theta_{0}}\right) \tilde{q}_{t}\right], \\
& N_{s}^{2}=g \frac{d}{d z}\left[\frac{\tilde{\theta}_{e}}{\theta_{0}}-\left(R_{v d}-\frac{L_{v}}{c_{p} \theta_{0}}+1\right) q_{v s}(z)-\tilde{q}_{t}\right] .
\end{aligned}
$$

Similar types of equations for nonprecipitating cloud dynamics have been considered in the past (e.g., 
Kuo 1961; Bretherton 1987; Grabowski and Clark 1993; Cuijpers and Duynkerke 1993; Stevens 2007; Pauluis and Schumacher 2010, 2011). A derivation of (8), starting from the perhaps more familiar equations for equivalent potential temperature $\theta_{e}$ and total water $q_{t}$, is described in the appendix.

\section{c. Piecewise quadratic energy}

In this section, we present one of the main results of the paper: we show that there is a piecewise quadratic, positive-definite conserved energy for the moist, nonprecipitating Boussinesq equations.

To find the piecewise quadratic energy, we take motivation from Smith and Stechmann (2017) and assume that the total energy, $E_{1}$, is the sum of four components: kinetic energy, unsaturated potential energy, saturated potential energy, and moist energy, which we denote as $\mathrm{KE}, \mathrm{PE}_{u}, \mathrm{PE}_{s}$, and $\mathrm{ME}$, respectively. Explicitly, this means that

$$
E_{1}=\mathrm{KE}+\mathrm{PE}_{u}+\mathrm{PE}_{s}+\mathrm{ME} .
$$

By analogy with Smith and Stechmann (2017), these components are assumed to take the form

$$
\begin{aligned}
\mathrm{KE} & =\frac{1}{2}|\mathbf{u}|^{2}, \\
\mathrm{PE}_{u} & =\frac{1}{2}\left(\frac{b_{u}^{2}}{N_{u}^{2}}\right) H_{u}, \\
\mathrm{PE}_{s} & =\frac{1}{2}\left(\frac{b_{s}^{2}}{N_{s}^{2}}\right) H_{s}, \\
\mathrm{ME} & =\frac{1}{2} A_{u} M^{2} H_{u}+\frac{1}{2} A_{s} M^{2} H_{s},
\end{aligned}
$$

where

$$
M=\frac{b_{u}}{N_{u}^{2}}-\frac{b_{s}}{N_{s}^{2}}
$$

and

$$
\frac{D M}{D t}=0
$$

so that $M$ is a material invariant.

To motivate the form we have chosen for the moist energy, ME, we make two observations. First, since $M$ is a material invariant, ME has no impact on energy transfers within each phase, but it can still impact energy transfers at the phase interface, due to the Heaviside factors in (13d). The coefficients $A_{u}$ and
$A_{s}$ are then determined to provide the appropriate amount of latent energy transferred at the interface. Second, the variable $M$ is associated with an additional eigenmode that arises for moist dynamics but is not present for dry dynamics. It essentially emerges from the linearization of (8) in the purely unsaturated or purely saturated case (see, e.g., Hernandez-Duenas et al. 2015; Smith and Stechmann 2017, and references therein). As such, it is natural to suppose that a moist energy should have an additional term related to $M^{2}$ and associated with this additional moist eigenmode. Indeed, other moist systems have also been shown to have an $M^{2}$ energy component (e.g., Frierson et al. 2004; Stechmann and Majda 2006; Chen and Stechmann 2016), but without the Heaviside factors that arise in ME in (13d) due to the phase changes.

The next step is to determine the values of $A_{u}$ and $A_{s}$, which are, as of yet, unknown constants. They will be determined by requiring that the material derivative of $E_{1}$ consist of only divergence terms. Following this idea, we differentiate each component of the total energy. The derivative of the kinetic energy is found by taking the dot product of (8a) with $\mathbf{u}$, and the derivative of $\mathrm{PE}_{u}$ is found by noting that

$$
\frac{D}{D t} \mathrm{PE}_{u}=\frac{D}{D t}\left(\frac{1}{2} \frac{b_{u}^{2}}{N_{u}^{2}}\right) H_{u}+\frac{1}{2} \frac{b_{u}^{2}}{N_{u}^{2}} \frac{D H_{u}}{D t} .
$$

The derivatives of $\mathrm{PE}_{s}$ and ME are determined similarly. We then have

$$
\begin{aligned}
\frac{D}{D t} \mathrm{KE} & =-\nabla \cdot(\phi \mathbf{u})+w b_{u} H_{u}+w b_{s} H_{s}, \\
\frac{D}{D t} \mathrm{PE}_{u} & =-w b_{u} H_{u}+\frac{1}{2} \frac{b_{u}^{2}}{N_{u}^{2}} \frac{D H_{u}}{D t}
\end{aligned}
$$

$$
\begin{aligned}
& \frac{D}{D t} \mathrm{PE}_{s}=-w b_{s} H_{s}+\frac{1}{2} \frac{b_{s}^{2}}{N_{s}^{2}} \frac{D H_{s}}{D t}, \\
& \frac{D}{D t} \mathrm{ME}=\frac{1}{2} A_{u} M^{2} \frac{D H_{u}}{D t}+\frac{1}{2} A_{s} M^{2} \frac{D H_{s}}{D t} .
\end{aligned}
$$

By looking at (16a)-(16d), we see that the saturated and unsaturated potential energies are involved with direct transfers to kinetic energy through the terms $-w b_{s} H_{s}$ and $-w b_{u} H_{u}$. In addition, the presence of the terms $D H_{u} / D t$, and $D H_{s} / D t$ indicate that they are also involved in exchanges with the moist energy at the phase interface. The role of the moist energy is to exchange energy with $\mathrm{PE}_{u}$ and $\mathrm{PE}_{s}$, and this exchange occurs only at the phase interface. The material derivative of the total energy is then 


$$
\begin{aligned}
\frac{D E_{1}}{D t}= & -\nabla \cdot(\phi \mathbf{u})+\frac{1}{2} \frac{b_{u}^{2}}{N_{u}^{2}} \frac{D H_{u}}{D t}+\frac{1}{2} \frac{b_{s}^{2}}{N_{s}^{2}} \frac{D H_{s}}{D t} \\
& +\frac{1}{2} A_{u} M^{2} \frac{D H_{u}}{D t}+\frac{1}{2} A_{s} M^{2} \frac{D H_{s}}{D t} .
\end{aligned}
$$

Note that the nondivergence terms are nonzero only at the phase interface. In fact, the moist energy ME is materially conserved at the phase interface, which indicates its role as a latent energy associated with phase changes.

To determine the values of $A_{u}$ and $A_{s}$, we require the nondivergence terms in (17) to vanish identically:

$$
\frac{1}{2} \frac{b_{u}^{2}}{N_{u}^{2}} \frac{D H_{u}}{D t}+\frac{1}{2} \frac{b_{s}^{2}}{N_{s}^{2}} \frac{D H_{s}}{D t}+\frac{1}{2} A_{u} M^{2} \frac{D H_{u}}{D t}+\frac{1}{2} A_{s} M^{2} \frac{D H_{s}}{D t}=0 .
$$

This is made easier by two observations. First, the interface flux terms $D H_{u} / D t$ and $D H_{s} / D t$ are nonzero only at the phase interface between saturated and unsaturated regions, and at this interface, $b_{u}=b_{s}$. Second, the relationship $H_{s}=1-H_{u}$ allows us to write $D H_{u} / D t=-\left(D H_{s} / D t\right)$. Therefore, the previous equation becomes

$$
\begin{aligned}
& {\left[-\frac{1}{2} A_{u}\left(\frac{1}{N_{u}^{2}}-\frac{1}{N_{s}^{2}}\right)^{2}-\frac{1}{2} \frac{1}{N_{u}^{2}}+\frac{1}{2} A_{s}\left(\frac{1}{N_{s}^{2}}-\frac{1}{N_{s}^{2}}\right)^{2}\right.} \\
& \left.+\frac{1}{2} \frac{1}{N_{s}^{2}}\right] b_{s}^{2} \frac{D H_{s}}{D t}=0 .
\end{aligned}
$$

The above equation yields the following relationship between $A_{u}$ and $A_{s}$ :

$$
A_{u}=A_{s}+\frac{N_{u}^{2} N_{s}^{2}}{N_{u}^{2}-N_{s}^{2}} .
$$

We thus have an infinite family of conserved energies which are quadratic even in the presence of phase changes, and differ only in the choice of $A_{u}$ and $A_{s}$. In addition, (20) implies that if $A_{s} \geq 0$, and if $N_{u}^{2}>N_{s}^{2}$, then the energy will be positive definite. Finally, letting $A_{s}=$ 0 is an appealing choice, since it allows $M$ to represent latent energy in the unsaturated phase that is transferred to buoyant potential energy upon reaching saturation; this choice results in the particularly simple expression

$$
\begin{aligned}
E_{1}= & \frac{1}{2} \mathbf{u}^{2}+\frac{1}{2}\left(\frac{b_{u}^{2}}{N_{u}^{2}}\right) H_{u}+\frac{1}{2}\left(\frac{b_{s}^{2}}{N_{s}^{2}}\right) H_{s} \\
& +\frac{1}{2}\left(\frac{N_{u}^{2} N_{s}^{2}}{N_{u}^{2}-N_{s}^{2}}\right) M^{2} H_{u},
\end{aligned}
$$

and satisfies the conservation equation

$$
\frac{\partial E_{1}}{\partial t}+\nabla \cdot\left[\mathbf{u}\left(E_{1}+\phi\right)\right]=0 .
$$

The energy $E_{1}$ in (21) is therefore the desired energy that is piecewise quadratic.

Several notes about the piecewise quadratic energy in (21) are in order. The first thing to point out is that we could absorb the factor of $\left(N_{u}^{2} N_{s}^{2}\right) /\left(N_{u}^{2}-N_{s}^{2}\right)$ into the definition of $M$ given in (14), and the resulting moist energy would then have the simpler form of $M^{2} H_{u} / 2$. Second, notice that, because it is (piecewise) quadratic, the energy in (21) can be used to define an inner product and a norm. Its use as a norm is explored below in section 5 for defining an energy for empirical orthogonal function analysis. Note, though, that (21) defines an inner product or norm only if the Heaviside functions $H_{u}$ and $H_{s}$ can be treated as given functions, as in some data analysis applications.

Finally, notice that $\mathrm{PE}_{u}, \mathrm{PE}_{s}$, and $\mathrm{ME}$ are all discontinuous across the phase interface, but the total potential energy, $\mathrm{PE}_{u}+\mathrm{PE}_{s}+\mathrm{ME}$, is continuous. To see this, note that the potential energy is $b_{u}^{2} /\left(2 N_{u}^{2}\right)+A_{u} M^{2} / 2$ in unsaturated regions and $b_{s}^{2} /\left(2 N_{s}^{2}\right)+A_{s} M^{2} / 2$ in saturated regions, which are both continuous functions of the variables $b_{u}$ and $b_{s}$ within the unsaturated and saturated phases, respectively. Thus, to establish continuity of the potential energy over the entire domain, all we need to do is verify that it is continuous at the phase interface. This can be expressed mathematically as $\lim _{b_{u} \rightarrow b_{s}} b_{u}^{2} /\left(2 N_{u}^{2}\right)+\left(A_{u} / 2\right) M^{2}-b_{s}^{2} /\left(2 N_{s}^{2}\right)-\left(A_{s} / 2\right) M^{2}=0$. To show that this limit is in fact zero, we substitute $b_{u}=b_{s}$, use (20), and simplify. The details are omitted for the sake of brevity.

Recall that the relationship between the coefficients $A_{u}$ and $A_{s}$ given by (20) was obtained by forcing the material derivative of the total energy to contain only divergence terms. This exact same relationship could have been obtained by instead demanding that the total energy be continuous across the phase interface.

Also note that $M^{2} H_{u}$ is similar in spirit to the moist latent energy $L_{v} q_{v}$ of a compressible atmosphere (e.g., Emanuel 1994), but also has some differences. It would be interesting to make a thorough comparison in the future, while for the moment we note the following comparison. For instance, they are similar in that they are both latent energies; that is, they are conserved quantities in unsaturated regions, and their energy can be accessed due to phase changes. They differ, however, in the release of their latent energy: the $M^{2} H_{u}$ energy is transferred to buoyant potential energy only at the phase interface, whereas $L_{v} q_{v}$ is a source of heating or cooling throughout the saturated region. 


\section{d. The traditional form of the potential energy}

Another version of the potential energy, one that is not piecewise quadratic, has also been considered in the past (e.g., Pauluis 2008; Hernandez-Duenas et al. 2013). Here we will write this traditional form in terms of the variables

$$
\begin{aligned}
& b_{u}^{\mathrm{tot}}=b_{u}+N_{u}^{2} z, \\
& b_{s}^{\mathrm{tot}}=b_{s}+N_{s}^{2} z,
\end{aligned}
$$

referred to as the total unsaturated and saturated buoyancies, respectively. These two variables are both material invariants, that is,

$$
\frac{D b_{u}^{\text {tot }}}{D t}=0, \quad \frac{D b_{s}^{\text {tot }}}{D t}=0 .
$$

The more traditional form of the potential energy, denoted by $\Pi$, is then given by

$\Pi\left(b_{u}^{\text {tot }}, b_{s}^{\text {tot }}, z\right)=-\int_{a}^{z}\left(b_{u}^{\text {tot }}-N_{u}^{2} z^{\prime}\right) H_{u}+\left(b_{s}^{\text {tot }}-N_{s}^{2} z^{\prime}\right) H_{s} d z^{\prime}$,

where $a$ is an arbitrary reference level, $H_{u}=$ $H\left[b_{u}^{\text {tot }}-b_{s}^{\text {tot }}-\left(N_{u}^{2}-N_{s}^{2}\right) z^{\prime}\right], H_{s}=1-H_{u}$, and the integration is performed while keeping $b_{u}^{\text {tot }}$ and $b_{s}^{\text {tot }}$ constant. The details of this integration are included in appendix $\mathrm{B}$, with the explicit form of $\Pi$ given by (B6). If we define a total energy $E_{2}$ as

$$
E_{2}=\frac{|\mathbf{u}|^{2}}{2}+\Pi
$$

then it can be shown that $E_{2}$ satisfies the equation

$$
\frac{\partial E_{2}}{\partial t}+\nabla \cdot\left\{\mathbf{u}\left[\left(E_{2}+\phi\right)\right]\right\}=0 .
$$

\section{e. Relating the two energies}

We have thus derived two conserved energies. The piecewise quadratic energy $E_{1}$ is shown above in (21), and the more traditional energy $E_{2}$ in terms of potential energy $\Pi$ is shown above in (26).

Here, we point out that the two energies are, in fact, related. In appendix B, it is shown that

$$
E_{1}=E_{2}+\Pi_{\mathrm{inv}},
$$

where $\Pi_{\text {inv }}$ denotes a material invariant term-that is, a term which satisfies $D \Pi_{\text {inv }} / D t=0$ and does not affect energy transfers. Thus, $E_{1}$ and $E_{2}$ differ by a material invariant. So, even after incorporating moisture and phase changes into the Boussinesq equations, we are still able to obtain a relationship between two energies that is similar to that of the dry case in (7).

\section{Anelastic equations}

We now study energetics of the anelastic equations. As above, the energy can be decomposed into four similar components $\left(\mathrm{KE}, \mathrm{PE}_{u}, \mathrm{PE}_{s}, \mathrm{ME}\right)$ with straightforward physical interpretations, but in this case the energy is not quadratic, since the coefficients $N_{u}^{2}(z)$ and $N_{s}^{2}(z)$ are not constants. The structure of this section is similar to that of the Boussinesq case; we will first consider the energetics for the dry equations, and then incorporate moisture and phase changes.

\section{a. Energetics for the dry anelastic equations}

The anelastic equations for a dry atmosphere with no phase changes are

$$
\begin{aligned}
\frac{D \mathbf{u}}{D t} & =-\nabla \phi+b \hat{\mathbf{z}}, \\
\frac{D b}{D t}+N^{2}(z) w & =0, \\
\nabla \cdot[\tilde{\rho}(z) \mathbf{u}] & =0,
\end{aligned}
$$

where $\phi=p^{\prime} / \tilde{\rho}(z)$ and $b$ is the buoyancy. In the anelastic case, the squared buoyancy frequency $N^{2}(z)$ will no longer be constant, which precludes the existence of a conservation equation like that of (6) for a quadratic energy. We can, however, still obtain such an equation for a slightly altered version of (2) that takes into account the varying nature of $N^{2}(z)$. Thus, we define,

$$
E_{2}^{\text {Anelastic }}=\frac{|\mathbf{u}|^{2}}{2}-\int_{a}^{z} b^{\text {tot }}-\tilde{b}\left(z^{\prime}\right) d z^{\prime},
$$

where the integral is performed as partial integration with $b^{\text {tot }}$ held fixed, and $\tilde{b}(z)=\int_{0}^{z} N^{2}(r) d r$, and $b^{\text {tot }}=$ $\tilde{b}(z)+b$ with $D b^{\text {tot }} / D t=0$. It can be shown that in the dry anelastic case we have

$$
\frac{\partial}{\partial t}\left[\tilde{\rho}(z) E_{2}^{\text {Anelastic }}\right]+\nabla \cdot\left[\tilde{\rho}(z) \mathbf{u}\left(E_{2}^{\text {Anelastic }}+\phi\right)\right]=0,
$$

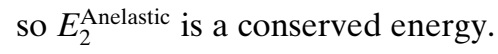

\section{b. Energetics for the anelastic equations with moisture and phase changes}

After incorporating moisture and phase changes, the anelastic equations are 


$$
\begin{aligned}
\frac{D \mathbf{u}}{D t} & =-\nabla \phi+\left(b_{u} H_{u}+b_{s} H_{s}\right) \hat{\mathbf{z}}, \\
\frac{D b_{u}}{D t}+N_{u}^{2}(z) w & =0, \\
\frac{D b_{s}}{D t}+N_{s}^{2}(z) w & =0, \\
\nabla \cdot[\tilde{\rho}(z) \mathbf{u}] & =0,
\end{aligned}
$$

where $\phi=p / \tilde{\rho}(z)$, and $\tilde{\rho}(z)$ is the background density profile.

The potential energy is again defined as the integral of the buoyancy:

$$
\begin{aligned}
\Pi\left(b_{u}^{\mathrm{tot}}, b_{s}^{\mathrm{tot}}, z\right) & =-\int_{a}^{z}\left[b_{u}^{\mathrm{tot}}-\tilde{b}_{u}\left(z^{\prime}\right)\right] H_{u} \\
& +\left[b_{s}^{\mathrm{tot}}-\tilde{b}_{s}\left(z^{\prime}\right)\right] H_{s} d z^{\prime},
\end{aligned}
$$

where the background states are defined as

$$
\begin{aligned}
& \tilde{b}_{u}(z)=\int_{0}^{z} N_{u}^{2}(r) d r, \\
& \tilde{b}_{s}(z)=\int_{0}^{z} N_{s}^{2}(r) d r .
\end{aligned}
$$

and the total variables are defined as

$$
\begin{gathered}
b_{u}^{\mathrm{tot}}=\tilde{b}_{u}(z)+b_{u}, \\
b_{s}^{\mathrm{tot}}=\tilde{b}_{s}(z)+b_{s},
\end{gathered}
$$

where $b_{u}^{\text {tot }}$ and $b_{s}^{\text {tot }}$ are material invariants: $D b_{u}^{\text {tot }} / D t=0$ and $D b_{s}^{\text {tot }} / D t=0$. Note that (35a) and (35b) are analogous to (23a) and (23b) in the Boussinesq case, and both definitions will agree when $N_{u}^{2}$ and $N_{s}^{2}$ are constant.

The main result of this subsection is a decomposition of the potential energy as

$$
\Pi=\Pi_{b_{u}}+\Pi_{b_{s}}+\Pi_{m}+\Pi_{\mathrm{inv}},
$$

where $\Pi_{b_{u}}, \Pi_{b_{s}}, \Pi_{m}$, and $\Pi_{\text {inv }}$, are the saturated potential energy, unsaturated potential energy, latent energy, and material invariant terms of $\Pi$, respectively. Upon integrating (33), we find that

$$
\begin{aligned}
& \Pi_{b_{u}}=-H_{u} \int_{a}^{z}\left[b_{u}^{\mathrm{tot}}-\tilde{b}_{u}\left(z^{\prime}\right)\right] d z^{\prime}, \\
& \Pi_{b_{s}}=-H_{s} \int_{a}^{z}\left[b_{s}^{\mathrm{tot}}-\tilde{b}_{s}\left(z^{\prime}\right)\right] d z^{\prime}, \\
& \Pi_{m}=H_{u} \int_{a}^{z_{r}}\left\{b_{u}^{\mathrm{tot}}-b_{s}^{\mathrm{tot}}-\left[\tilde{b}_{u}\left(z^{\prime}\right)-\tilde{b}_{s}\left(z^{\prime}\right)\right]\right\} d z^{\prime},
\end{aligned}
$$

and $\Pi_{\text {inv }}$ is a material invariant that satisfies $D \Pi_{\text {inv }} / D t=$ 0 . The calculations leading from (33) to (37) are similar to the ones done in the appendixes and can be found in the online supplemental material. In brief, the terms $\Pi_{b_{u}}$ and $\Pi_{b_{s}}$ are similar to the potential energy in the dry case, except they are multiplied by Heaviside functions $H_{u}$ and $H_{s}$, respectively, in this case with phase changes; and the third term $\Pi_{m}$ represents an additional moist latent energy.

Note that $z_{r}$, not $z$, appears as the upper limit of integration in (37c), where $z_{r}$ is the solution to the equation

$$
b_{u}^{\text {tot }}-b_{s}^{\text {tot }}=\tilde{b}_{u}\left(z_{r}\right)-\tilde{b}_{s}\left(z_{r}\right),
$$

where $b_{u}^{\text {tot }}$ and $b_{s}^{\text {tot }}$ are regarded as fixed values. Intuitively, $z_{r}$ is essentially a lifted condensation level (LCL), since (38) defines $z_{r}$ as the level where the unsaturated and saturated buoyancies, $b_{u}$ and $b_{s}$, are equal, which is the condition for saturation. Also notice that $z_{r}$ is a material invariant, since it is a function of the material invariants $b_{u}^{\text {tot }}$ and $b_{s}^{\text {tot }}$, and therefore $z_{r}$ satisfies $D z_{r} / D t=0$.

Also notice that $\Pi_{m}$ in (37c) is analogous to the moist latent energy $\propto M^{2} H_{u}$ in the Boussinesq case in (21). As such, (37c) could possibly be used as a definition of a variable like $M$ in the anelastic case.

To see the different roles of the components of $\Pi$, one can examine the energy transfers:

$$
\frac{D \Pi_{b_{u}}}{D t}=-b_{u} H_{u} w-\left\{\int_{a}^{z}\left[b_{u}^{\mathrm{tot}}-\tilde{b}_{u}\left(z^{\prime}\right)\right] d z^{\prime}\right\} \frac{D H_{u}}{D t},
$$

$$
\frac{D \Pi_{b_{s}}}{D t}=-b_{s} H_{s} w-\left\{\int_{a}^{z}\left[b_{s}^{\text {tot }}-\tilde{b}_{s}\left(z^{\prime}\right)\right] d z^{\prime}\right\} \frac{D H_{s}}{D t},
$$

$$
\frac{D \Pi_{m}}{D t}=\left(\int_{a}^{z_{r}}\left\{b_{u}^{\mathrm{tot}}-b_{s}^{\mathrm{tot}}-\left[\tilde{b}_{u}\left(z^{\prime}\right)-\tilde{b}_{s}\left(z^{\prime}\right)\right]\right\} d z^{\prime}\right) \frac{D H_{u}}{D t},
$$

$\frac{D \Pi_{\text {inv }}}{D t}=0$.

Notice that the only terms that are involved in direct transfers of energy with kinetic energy are the terms $\Pi_{b_{u}}$, and $\Pi_{b_{s}}$. The term $\Pi_{\text {inv }}$ is a material invariant, and $\Pi_{m}$, which is given by (37c), transfers energy only at the phase interface. Furthermore, by combining (39a)-(39c), one can see that

$$
\frac{D}{D t}\left(\Pi_{b_{u}}+\Pi_{b_{s}}+\Pi_{m}\right)=-w b,
$$

where we have also used that $z_{r}$ is equal to $z$ at the phase interface. Hence, the material derivative of $\Pi_{m}$ is precisely 
what cancels the interface flux terms that arise in the flux of the buoyant potential energy terms in (39a) and (39b). So from this perspective, $\Pi_{m}$ is analogous to the moist energy of (13d) in that both terms ensure that the total potential energy is continuous, by supplying moist latent energy at the phase interface. In addition, it can be shown that

$$
\frac{\partial}{\partial t}\left[\tilde{\rho}(z)\left(\frac{|\mathbf{u}|^{2}}{2}+\Pi\right)\right]+\nabla \cdot\left[\tilde{\rho}(z) \mathbf{u}\left(\frac{|\mathbf{u}|^{2}}{2}+\Pi+\phi\right)\right]=0
$$

so that the total energy is conserved.

\section{c. Alternative energy, anelastic $M$, and connection with Boussinesq case}

We end this section by offering a slightly different definition of $\Pi$. The alternative $\Pi$ will serve two purposes: (i) to make a connection with the Boussinesq case, and (ii) to motivate a definition of an $M$ variable in the anelastic case, and to offer a physical interpretation of that $M$.

To these ends, we define $\Pi^{*}$ as

$$
\begin{aligned}
\Pi^{*}\left(b_{u}^{\mathrm{tot}}, b_{s}^{\mathrm{tot}}, z\right)= & -\int_{a_{1}}^{z}\left[b_{u}^{\mathrm{tot}}-\tilde{b}_{u}\left(z^{\prime}\right)\right] H_{u} d z^{\prime} \\
& -\int_{a_{2}}^{z}\left[b_{s}^{\mathrm{tot}}-\tilde{b}_{s}\left(z^{\prime}\right)\right] H_{s} d z^{\prime},
\end{aligned}
$$

where $a_{1}$ and $a_{2}$ are reference heights. We note that $\Pi^{*}$ differs from $\Pi$ given by (33) by a material invariant (which can be seen by splitting the integral from $a_{1}$ to $z$ into two integrals, one from $a_{1}$ to $a$ and another from $a$ to $z$, and similarly for $a_{2}$ ). A convenient and physically relevant choice (as discussed further below) is to define $a_{1}=\tilde{b}_{u}^{-1}\left(b_{u}^{\text {tot }}\right)$ and $a_{2}=\tilde{b}_{s}^{-1}\left(b_{s}^{\text {tot }}\right)$, so that $a_{1}$ and $a_{2}$ correspond to the unsaturated and saturated levels of neutral buoyancy, respectively. Then, as was done for $\Pi$, we can decompose $\Pi^{*}$ into its unsaturated, saturated, latent, and invariant components, which are denoted by $\Pi_{b_{u}}^{*}, \Pi_{b_{s}}^{*}, \Pi_{m}^{*}$, and $\Pi_{\text {inv }}^{*}$, respectively.

In the special case of Boussinesq, where $N_{u}^{2}$ and $N_{s}^{2}$ are constants, the expressions for $\Pi_{b_{u}}^{*}, \Pi_{b_{s}}^{*}$, and $\Pi_{m}^{*}$ simplify to the quadratic unsaturated, saturated, and moist energies given in (21). In other words, we have, in the Boussinesq case,

$$
\begin{aligned}
& \Pi_{b_{u}}^{*}=\frac{1}{2}\left(\frac{b_{u}^{2}}{N_{u}^{2}}\right) H_{u}, \\
& \Pi_{b_{s}}^{*}=\frac{1}{2}\left(\frac{b_{s}^{2}}{N_{s}^{2}}\right) H_{s},
\end{aligned}
$$

$$
\Pi_{m}^{*}=\frac{1}{2}\left(\frac{N_{u}^{2} N_{s}^{2}}{N_{u}^{2}-N_{s}^{2}}\right) M^{2} H_{u} .
$$

We note that this connection with the Boussinesq case arises only for the specific choice of $a_{1}$ and $a_{2}$ as levels of neutral buoyancy as described above. If different values of $a_{1}$ and $a_{2}$ are used, or if the earlier $\Pi$ from (33) is used instead of the alternative $\Pi^{*}$ from (42), then the connection with the Boussinesq case is somewhat spoiled and (43) would have additional terms on the right-hand side.

Finally, we consider the question: How can an $M$ variable be defined in the anelastic case? In particular, note that we did not earlier define an $M$ variable in the anelastic case, whereas in the Boussinesq case we had defined $M$ before describing the energetics. The formula in (43c) now suggests a definition of the $M$ variable in the anelastic case, since

$$
\Pi_{m}^{*}=H_{u}\left\{\int_{a_{1}}^{z_{r}}\left[b_{u}^{\mathrm{tot}}-\tilde{b}_{u}\left(z^{\prime}\right)\right] d z^{\prime}-\int_{a_{2}}^{z_{r}}\left[b_{s}^{\mathrm{tot}}-\tilde{b}_{s}\left(z^{\prime}\right)\right] d z^{\prime}\right\} .
$$

By comparing (43c) and (44), one is motivated to define

$$
\begin{aligned}
M_{\text {anelastic }}= & \left\{\int_{a_{1}}^{z_{r}}\left[b_{u}^{\text {tot }}-\tilde{b}_{u}\left(z^{\prime}\right)\right] d z^{\prime}\right. \\
& \left.-\int_{a_{2}}^{z_{r}}\left[b_{s}^{\text {tot }}-\tilde{b}_{s}\left(z^{\prime}\right)\right] d z^{\prime}\right\}^{1 / 2},
\end{aligned}
$$

since it would be proportional to the Boussinesq definition of $M$ in the special case of constant $N_{u}^{2}$ and $N_{s}^{2}$. An alternative definition of an anelastic $M$ variable was proposed by Wetzel et al. (2019). An advantageous property of $M_{\text {aneastic }}$ here is its physical interpretation: it is related to convective available potential energy (CAPE) (e.g., Moncrieff and Miller 1976; Emanuel 1994; Hernandez-Duenas et al. 2019). In particular, notice that (45) involves the difference between an unsaturated CAPE and a saturated CAPE, since $z_{r}$ is similar to a lifted condensation level [see its definition in (38)] and $a_{1}$ and $a_{2}$ are levels of neutral buoyancy. It would be interesting to investigate the variable $M_{\text {aneastic }}$ in more thorough detail in the future.

\section{Other moist systems}

In this section, we will examine several systems featuring other microphysical parameterizations. We will start by looking at energetics of the anelastic equations when Kessler microphysics are used. The Kessler case 
allows an exploration of the effects of an additional water constituent, rainwater, and associated source terms and their influence on energetics. Then a limiting form of the Kessler scheme will be considered, called the FARE microphysics scheme (Hernandez-Duenas et al. 2013). The FARE case is interesting because it includes rainwater and precipitation, yet, unlike the Kessler case, it has an energy that is conserved (aside from a single dissipation term, due to precipitation at the surface).

\section{a. Warm-rain microphysics}

The anelastic equations with warm-rain microphysics can be used to model a moist atmosphere with three phases of water: cloud water $q_{c}$, rainwater $q_{r}$, and water vapor $q_{v}$ (Kessler 1969; Grabowski and Smolarkiewicz 1996). They are

$$
\begin{gathered}
\frac{D \mathbf{u}}{D t}=-\nabla \phi+\left(b_{u} H_{u}+b_{s} H_{s}\right) \hat{z}, \\
\frac{D b_{u}}{D t}+N_{u}^{2}(z) w-\frac{g}{\tilde{\rho}(z)}\left(R_{v d}-\frac{L_{v}}{c_{p} \theta_{0}}\right) \frac{\partial}{\partial z}\left[\tilde{\rho}(z) V_{T} q_{r}\right] \\
=-g\left(R_{v d}-\frac{L_{v}}{c_{p} \theta_{0}}+1\right)\left(A_{r}+C_{r}-E_{r}\right), \\
\frac{D b_{s}}{D t}+N_{s}^{2}(z) w+\frac{g}{\tilde{\rho}(z)} \frac{\partial}{\partial z}\left[\tilde{\rho}(z) V_{T} q_{r}\right]=0,
\end{gathered}
$$

$$
\begin{aligned}
\frac{D q_{r}}{D t}-\frac{1}{\tilde{\rho}(z)} \frac{\partial}{\partial z}\left[\tilde{\rho}(z) V_{T} q_{r}\right] & =A_{r}+C_{r}-E_{r}, \\
\nabla \cdot[\tilde{\rho}(z) \mathbf{u}] & =0,
\end{aligned}
$$

where $A_{r}$ is the autoconversion of cloud water into rainwater, $C_{r}$ is the collection of cloud water by rain, and $E_{r}$ is the evaporation of rainwater into water vapor, $V_{T}$ is the rainfall velocity, and we have assumed that supersaturation cannot occur, and again $\phi=p / \tilde{\rho}(z)$. Many other formulations of cloud microphysics are in use, such as simple formulations (e.g., Vallis et al. 2019) and more complex formulations including number concentrations of droplets and/or ice microphysics (e.g., Seifert and Beheng 2001, 2006); it would be interesting to analyze these or other formulations, but we focus on the Kessler system here as an example case. Additional details regarding the Kessler scheme can be found in appendix A.

Now consider energetics, and take the potential energy $\Pi$ to have the same definition as in the nonprecipitating anelastic case from (33). Despite the increased microphysical complexity of the Kessler system, we will still obtain the exact same expression for $\Pi$ when it is integrated. That is, $\Pi$ can still be integrated and decomposed as in (36)-(37). The energy evolution equation satisfied by $\Pi$ in the Kessler scheme will, however, differ from the one in the purely anelastic case. Here, it takes the form

$$
\begin{aligned}
\frac{\partial}{\partial t}\left[\tilde{\rho}(z)\left(\frac{|\mathbf{u}|^{2}}{2}+\Pi\right)\right]+\nabla \cdot\left[\tilde{\rho}(z) \mathbf{u}\left(\frac{|\mathbf{u}|^{2}}{2}+\Pi+\phi\right)\right]= & g(z-a) \frac{\partial}{\partial z}\left[V_{T} \tilde{\rho}(z) q_{r}\right]-g\left(z-z_{r}\right)\left(R_{v d}-\frac{L_{v}}{c_{p} \theta_{0}}+1\right) \\
& \times\left\{\frac{\partial}{\partial z}\left[V_{T} \tilde{\rho}(z) q_{r}\right]-\tilde{\rho}(z) S_{r}\right\} H_{u}-g\left(z_{r}-a\right)\left(R_{v d}-\frac{L_{v}}{c_{p} \theta_{0}}+1\right) \\
& \times\left\{\frac{\partial}{\partial z}\left[V_{T} \tilde{\rho}(z) q_{r}\right]-\tilde{\rho}(z) S_{r}\right\} H\left[b_{u}^{\text {tot }}-b_{s}^{\text {tot }}-\left(\tilde{b}_{u}-\tilde{b}_{s}\right)(a)\right]
\end{aligned}
$$

where $S_{r}=A_{r}+C_{r}-E_{r}$. The reason for the terms on the right-hand side of (47) is that $b_{u}$ and $b_{s}$ are not material invariants, and so their derivatives will appear when we differentiate $\Pi$. As a result, an energy principle can be written down for the case of Kessler microphysics, but the energy is not conserved, due to rain falling at velocity $V_{T}$ and due to microphysical source terms $S_{r}=A_{r}+C_{r}-E_{r}$.

\section{b. FARE microphysics}

In this section, we consider energetics for a system that includes precipitation yet still has a piecewise quadratic energy, and the energy is conserved (aside from a single dissipation term, due to precipitation at the surface).

The system of interest here is the fast autoconversion and rain evaporation model, or FARE model, first introduced by Hernandez-Duenas et al. (2013). This model can be obtained from the anelastic Kessler model by making several assumptions. First, we assume that water vapor is converted directly into rainwater. This obviates the need for a $q_{c}$ variable as well as expressions for $A_{r}$ and $C_{r}$, and constitutes the fast autoconversion 
assumption. Second, rainwater, which is assumed to fall at a constant rate $V_{T}$, is instantly evaporated in unsaturated regions until saturation is reached. This amounts to the fast rain evaporation assumption and completes a basic description of the FARE model, the equations of which are

$$
\begin{aligned}
& \frac{D \mathbf{u}}{D t}=-\nabla \phi+\left(b_{u} H_{u}+b_{s} H_{s}\right) \hat{\mathbf{z}}, \\
& \frac{D b_{u}}{D t}+N_{u}^{2} w-g V_{T}\left(R_{v d}-\frac{L_{v}}{c_{p} \theta_{0}}\right) \frac{\partial q_{r}}{\partial z}=0, \\
& \frac{D b_{s}}{D t}+N_{s}^{2} w+g V_{T} \frac{\partial q_{r}}{\partial z}=0, \\
& \nabla \cdot \mathbf{u}=0 .
\end{aligned}
$$

Note that these equations are essentially the same as the nonprecipitating Boussinesq equations from section $2 \mathrm{~b}$, except additional $V_{T}$ terms are present and represent precipitation.

Recall that in section $2 b$, we found an infinite family of conserved, quadratic energies of the moist, nonprecipitating Boussinesq equations of $(8 \mathrm{a})-(8 \mathrm{~d})$. We now show that by including the microphysics of the FARE model, that is, by incorporating a nonzero value of $V_{T}$, we can obtain a unique quadratic energy, which under appropriate circumstances, will be positive definite.

Our starting point, then, is the energy defined by (13a)-(13d), and (20), and the goal is to determine values for the constants $A_{u}$ and $A_{s}$ that appear in the energy. Differentiating each component of the energy under these new microphysical assumptions now results in

$$
\begin{aligned}
\frac{D}{D t} \mathrm{KE}= & -\nabla \cdot(\phi \mathbf{u})+w b_{u} H_{u}+w b_{s} H_{s}, \\
\frac{D}{D t} \mathrm{PE}_{u}= & -w b_{u} H_{u}+\frac{b_{u} g V_{T}}{N_{u}^{2}}\left(R_{v d}-\frac{L_{v}}{c_{p} \theta_{0}}\right) \frac{\partial q_{r}}{\partial z} H_{u} \\
& +\frac{1}{2} \frac{b_{u}^{2}}{N_{u}^{2}} \frac{D H_{u}}{D t}, \\
\frac{D}{D t} \mathrm{PE}_{s}= & -w b_{s} H_{s}-\frac{b_{s} g V_{T}}{N_{s}^{2}} \frac{\partial q_{r}}{\partial z} H_{s}+\frac{1}{2} \frac{b_{s}^{2}}{N_{S}^{2}} \frac{D H_{s}}{D t}, \\
\frac{D}{D t} \mathrm{ME}= & A_{u} M \frac{D M}{D t} H_{u}+\frac{1}{2} A_{u} M^{2} \frac{D H_{u}}{D t}+A_{s} M \frac{D M}{D t} H_{s} \\
& +\frac{1}{2} A_{s} M^{2} \frac{D H_{s}}{D t},
\end{aligned}
$$

where

$$
\frac{D M}{D t}=g V_{T}\left[\frac{1}{N_{u}^{2}}\left(R_{v d}-\frac{L_{v}}{c_{p} \theta_{0}}\right)+\frac{1}{N_{s}^{2}}\right] \frac{\partial q_{r}}{\partial z} .
$$

Since there is no rainfall in unsaturated regions in the FARE model, it follows that $\left(\partial q_{r} / \partial z\right) H_{u}=0$. Using this, along with the relationship between $A_{u}$ and $A_{s}$ given by (20), we can write the material derivative of the total energy as

$$
\begin{aligned}
\frac{D E_{1}}{D t}= & -\nabla \cdot(\phi \mathbf{u})-\frac{b_{s} g V_{T}}{N_{s}^{2}} \frac{\partial q_{r}}{\partial z} H_{s} \\
& +A_{s} M g V_{T}\left[\frac{1}{N_{u}^{2}}\left(R_{v d}-\frac{L_{v}}{c_{p} \theta_{0}}\right)+\frac{1}{N_{s}^{2}}\right] \frac{\partial q_{r}}{\partial z} H_{s},
\end{aligned}
$$

which appears to have nondivergence terms on the righthand side.

Now we can determine the unique value of $A_{s}$ such that (51) contains only divergence terms. This can be accomplished by rewriting that equation in terms of $M$ and $q_{r}$. In particular, the goal is to write the $b_{s} \partial q_{r} / \partial z$ term as a sum of a $q_{r} \partial q_{r} / \partial z$ term and a $M \partial q_{r} / \partial z$ term, and then to choose $A_{s}$ to make the $M \partial q_{r} / \partial z$ term vanish. To this end, observing that

$$
q_{r}=\frac{1}{g\left(R_{v d}-\frac{L_{v}}{c_{p} \theta_{0}}+1\right)}\left(b_{u}-b_{s}\right) H_{s}
$$

and

$$
b_{s} H_{s}=\frac{N_{s}^{2} g V_{T}\left(R_{v d}-\frac{L_{v}}{c_{p} \theta_{0}}+1\right)}{N_{u}^{2}-N_{s}^{2}} q_{r}-\frac{N_{u}^{2} N_{s}^{2}}{N_{u}^{2}-N_{s}^{2}} M H_{s},
$$

we can simplify (51) into

$$
\begin{aligned}
\frac{D E}{D t}= & -g^{2} V_{T}\left(\frac{R_{v d}-\frac{L_{v}}{c_{p} \theta_{0}}+1}{N_{u}^{2}-N_{s}^{2}} \frac{\partial q_{r}}{\partial z}\right) q_{r} \\
& +g V_{T} \frac{N_{u}^{2}}{N_{u}^{2}-N_{s}^{2}} M \frac{\partial q_{r}}{\partial z} H_{s} \\
& +A_{s} M g V_{T}\left[\frac{1}{N_{u}^{2}}\left(R_{v d}-\frac{L_{v}}{c_{p} \theta_{0}}\right)+\frac{1}{N_{s}^{2}}\right] \frac{\partial q_{r}}{\partial z} H_{s} .
\end{aligned}
$$

We can now solve for $A_{s}$ from the constraint that

$$
\begin{aligned}
& g V_{T} \frac{N_{u}^{2}}{N_{u}^{2}-N_{s}^{2}} M \frac{\partial q_{r}}{\partial z} H_{s}+A_{s} M g V_{T} \\
& \quad \times\left[\frac{1}{N_{u}^{2}}\left(R_{v d}-\frac{L_{v}}{c_{p} \theta_{0}}\right)+\frac{1}{N_{s}^{2}}\right] \frac{\partial q_{r}}{\partial z} H_{s}=0 .
\end{aligned}
$$


Doing so, we find that

$$
A_{s}=\frac{-N_{u}^{2}}{\left(N_{u}^{2}-N_{s}^{2}\right)\left[\frac{1}{N_{u}^{2}}\left(R_{v d}-\frac{L_{v}}{c_{p} \theta_{0}}\right)+\frac{1}{N_{s}^{2}}\right]} .
$$

Then, from (20), we have

$$
A_{u}=\frac{N_{s}^{2}\left(R_{v d}-\frac{L_{v}}{c_{p} \theta_{0}}\right)}{\left(N_{u}^{2}-N_{s}^{2}\right)\left[\frac{1}{N_{u}^{2}}\left(R_{v d}-\frac{L_{v}}{c_{p} \theta_{0}}\right)+\frac{1}{N_{s}^{2}}\right]} .
$$

With these values of $A_{u}$ and $A_{s}$, the total energy $E$ is given by

$$
\begin{aligned}
E= & \frac{1}{2}|\mathbf{u}|^{2}+\frac{1}{2}\left(\frac{b_{u}^{2}}{N_{u}^{2}}\right) H_{u}+\frac{1}{2}\left(\frac{b_{s}^{2}}{N_{s}^{2}}\right) H_{s} \\
& +\frac{1}{2} A_{u} M^{2} H_{u}+\frac{1}{2} A_{s} M^{2} H_{s},
\end{aligned}
$$

which completes the derivation of a piecewise quadratic energy.

Note from (56) and (57) that the coefficients $A_{u}$ and $A_{s}$ will be positive if $\left(N_{u}^{2} / N_{s}^{2}\right)<\left[\left(L_{v} / c_{p} \theta_{0}\right)-R_{v d}\right]$. We know that $R_{v d} \approx$ 0.61 and $L_{v} /\left(c_{p} \theta_{0}\right)=O(10)$, so the ratio $N_{u}^{2} / N_{s}^{2}$ can be roughly as large as $O(10)$, and so the condition on the ratio of the squared buoyancy frequencies to ensure positivity of $A_{u}$ and $A_{s}$ allows a reasonably wide range of values.

The conservation law for the energy in (58) takes the form

$$
\frac{\partial E}{\partial t}+\nabla \cdot[\mathbf{u}(E+\phi)]+\frac{1}{2} g^{2} V_{T}\left(\frac{R_{v d}-\frac{L_{v}}{c_{p} \theta_{0}}+1}{N_{u}^{2}-N_{s}^{2}}\right) \frac{\partial}{\partial z} q_{r}^{2}=0
$$

Thus, the effect of precipitation is an energy flux proportional to $V_{T} q_{r}^{2}$. Upon integrating this conservation law in space, one can see that the $V_{T} q_{r}^{2}$ term represents a sign-definite sink of energy at the lower boundary of the domain. In other words, when precipitation falls to the surface, it represents a loss of water and also a loss of energy from the system.

\section{Application to EOF analysis}

In this section, we use the ideas from the piecewisequadratic energy and apply them to empirical orthogonal function (EOF) analysis, or principal component analysis (PCA). In the EOF analysis, the goal is to decompose a signal (in our case, a variable of an atmospheric flow) into a set of spatial and temporal patterns, and to identify the patterns with the largest energy or variance. In its most common form, an EOF analysis would use the standard $L^{2}$ energy. Here, the goal is to see if we can use the concepts of the piecewise-quadratic energy from (21), in order to include information about phase changes, to potentially better identify the main modes of variability of moist dynamics.

We begin with a brief review of EOF methods. In EOF analysis, we assume that we have $N_{T}$ time samples of a scalar variable, $S(\mathbf{x}, t)$, located at $N$ spatial points. We denote the $N$ values of $S$ at time $t_{i}$ by the vector

$$
\mathbf{s}^{i}=\left(s_{1}^{i}, s_{2}^{i}, \ldots, s_{N}^{i}\right)^{\mathrm{T}} .
$$

The data matrix $\mathbf{S}$ is then formed by the column vectors $\mathbf{s}^{i}$ :

$$
\mathbf{S}=\left(\mathbf{s}^{1}, \mathbf{s}^{2}, \ldots, \mathbf{s}^{N_{T}}\right) .
$$

The EOF modes are then the eigenvectors of the sample covariance matrix $\left(1 / N_{T}\right) \mathbf{S S}^{\mathrm{T}}$.

To motivate the variables on which to perform the EOF analysis, recall from (21) that the energy

$$
E_{1}=\frac{1}{2}|\mathbf{u}|^{2}+\frac{1}{2}\left(\frac{b_{u}^{2}}{N_{u}^{2}}\right) H_{u}+\frac{1}{2}\left(\frac{b_{s}^{2}}{N_{s}^{2}}\right) H_{s}+\frac{1}{2} \frac{N_{u}^{2} N_{s}^{2}}{N_{u}^{2}-N_{s}^{2}} M^{2} H_{u}
$$

is a piecewise quadratic, positive definite conserved quantity, and it induces a natural inner product (assuming the values of the Heaviside functions $H_{u}(\mathbf{x}, t)$ and $H_{s}(\mathbf{x}, t)$ can be taken as given values, as in the case of the present data analysis application). While the EOF analysis below will deviate from using the full form of this energy, we explain the deviations further below and momentarily describe the full energy to aid the discussion. Consider the full state vector $\mathbf{y}$, where

$$
\mathbf{y}=\left(u, v, w, B, M^{*}\right),
$$

where

$$
B=\frac{b_{u}}{N_{u}} H_{u}+\frac{b_{s}}{N_{s}} H_{s}
$$

and

$$
M^{*}=M H_{u}
$$

Then (62) can be written as $E_{1}=\mathbf{y}^{\mathrm{T}} \mathbf{A y}$, where 


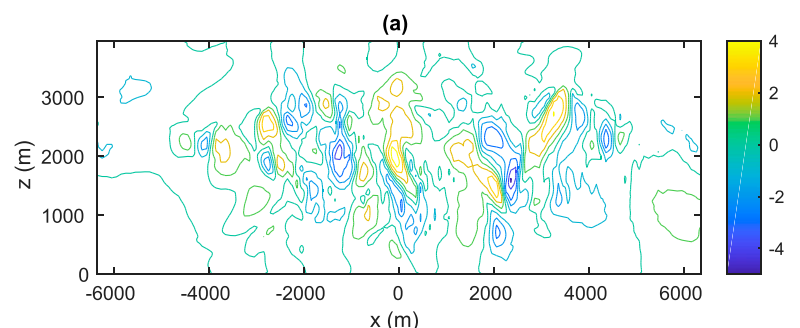

(b)

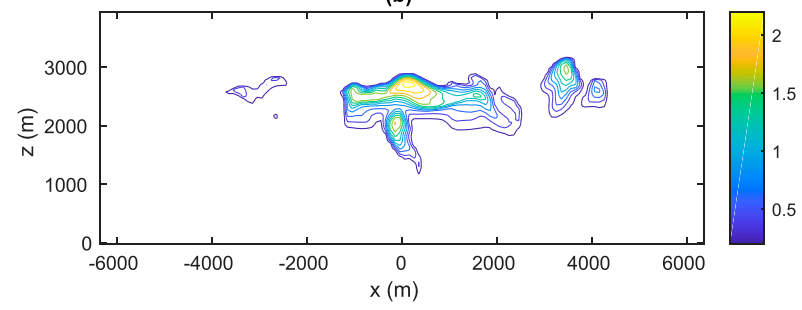

FIG. 1. Snapshots of the (top) vertical velocity $w\left(\mathrm{~m} \mathrm{~s}^{-1}\right)$ and (bottom) cloud water $q_{c}\left(\mathrm{~g} \mathrm{~kg}^{-1}\right)$ to illustrate the spatial structures present in the shallow convection simulation data used in the EOF analysis.

$$
\mathbf{A}=\frac{1}{2}\left(\begin{array}{ccccc}
1 & 0 & 0 & 0 & 0 \\
0 & 1 & 0 & 0 & 0 \\
0 & 0 & 1 & 0 & 0 \\
0 & 0 & 0 & 1 & 0 \\
0 & 0 & 0 & 0 & \frac{N_{u}^{2} N_{s}^{2}}{N_{u}^{2}-N_{s}^{2}}
\end{array}\right) .
$$

In writing $E_{1}=\mathbf{y}^{\mathrm{T}} \mathbf{A y}$, we have assumed that $B^{2}=$ $\left(b_{u}^{2} / b_{u}^{2}\right) H_{u}+\left(b_{s}^{2} / N_{s}^{2}\right) H_{s}$, which is valid if $H_{u}^{2}=H_{u}$, $H_{s}^{2}=H_{s}$, and $H_{u} H_{s}=0$. From the standpoint of data analysis, this means that all of our data points are away from the phase interface. In what follows, to examine the effect of phase changes in the simplest possible setting, we will consider the univariate case rather than the case of the full vector $\mathbf{y}$, and we will take the single variable to be either $B$ (as a setup with phase changes) or $b_{u}$ (as a comparison case with standard $L^{2}$ energy without phase changes).

We apply EOF analysis to simulations generated by the University of California, Los Angeles, large-eddy simulation (UCLA-LES) model based on data from the Rain in Cumulus Over the Ocean (RICO) study (Stevens et al. 2005; Rauber et al. 2007; Stechmann 2014). The focus of this study is on shallow cumulus clouds, which are clouds that extend only a few kilometers above Earth's surface. While these clouds do not involve ice, and often do not rain, they nevertheless incorporate both moisture and phase changes. The simulation is for 5 days with $N_{T}=7195$ time samples. For spatial resolution, we use a two-dimensional setup with $N_{x}=128$ and $N_{z}=100$ grid points with grid spacings of 100 and $40 \mathrm{~m}$ in
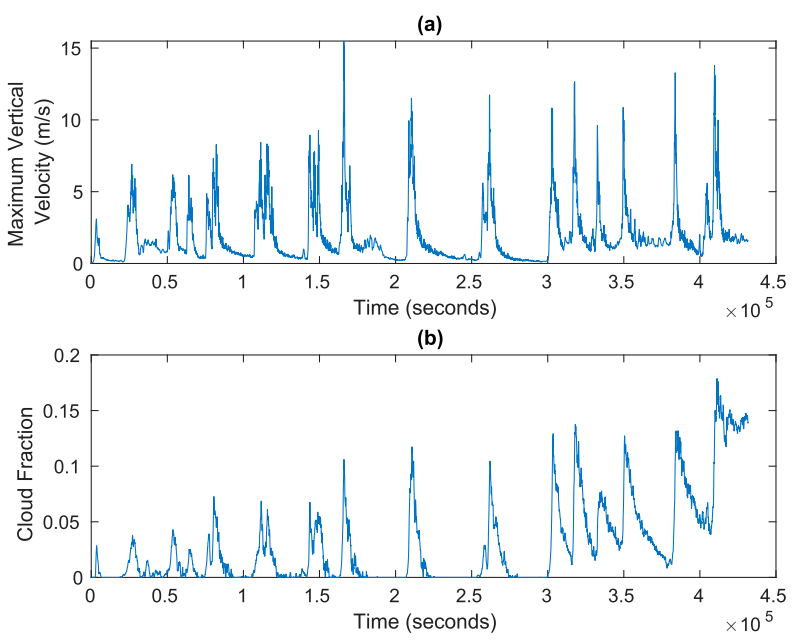

FIG. 2. Time series of the (top) maximum vertical velocity and (bottom) area cloud fraction to illustrate the intermittent evolution in time of the shallow convection simulation data used in the EOF analysis.

the horizontal and vertical directions, respectively. In Fig. 1, snapshots of the vertical velocity and cloud coverage are shown at approximately 2.4 days. (See Fig. 2 for a time series of vertical velocity and cloud fraction.)

Several additional steps are used in carrying out our analysis. First, to obtain enough data to ensure smoothness of the resulting EOFs, we perform an ensemble of simulations. Each ensemble member uses a different initial condition as random temperature perturbations are inserted below $200 \mathrm{~m}$, with a different random seed used for each member. Extending the method of EOF analysis on data produced from a single simulation to data from an ensemble is straightforward. To that end, suppose we have an ensemble with $N_{e}$ members, and denote the $k$ th member by $S^{k}(\mathbf{x}, t)$. The value of $S^{k}$ at time $t_{i}$ and location $x_{j}$ is denoted by

$$
\mathbf{s}^{i, k}=\left(s_{1}^{i, k}, s_{2}^{i, k}, \ldots, s_{N}^{i, k}\right)^{\mathrm{T}} .
$$

The data matrix of ensemble member $k$ is then given by

$$
\mathbf{S}^{k}=\left(\mathbf{s}^{1, k}, \mathbf{s}^{2, k}, \ldots, \mathbf{s}^{N_{T}, k}\right),
$$

and the corresponding ensemble covariance matrix $\mathbf{C}_{e}$ is

$$
\mathbf{C}_{e}=\frac{1}{N_{T}} \sum_{k=1}^{N_{e}}\left(\mathbf{S}^{k}\right)\left(\mathbf{S}^{k}\right)^{\mathrm{T}} .
$$

Second, we use deviations from the horizontal averages of $B$ and $b_{u}$ as our variables. That is to say, we subtract off the quantities $\left(1 / L_{x}\right) \int B\left(x^{\prime}, z, t\right) d x^{\prime}$ and $\left(1 / L_{x}\right) \int b_{u}\left(x^{\prime}, z, t\right) d x^{\prime}$ from $B$ and $b_{u}$, respectively, where $L_{x}$ denotes the horizontal domain length. Hence the data 


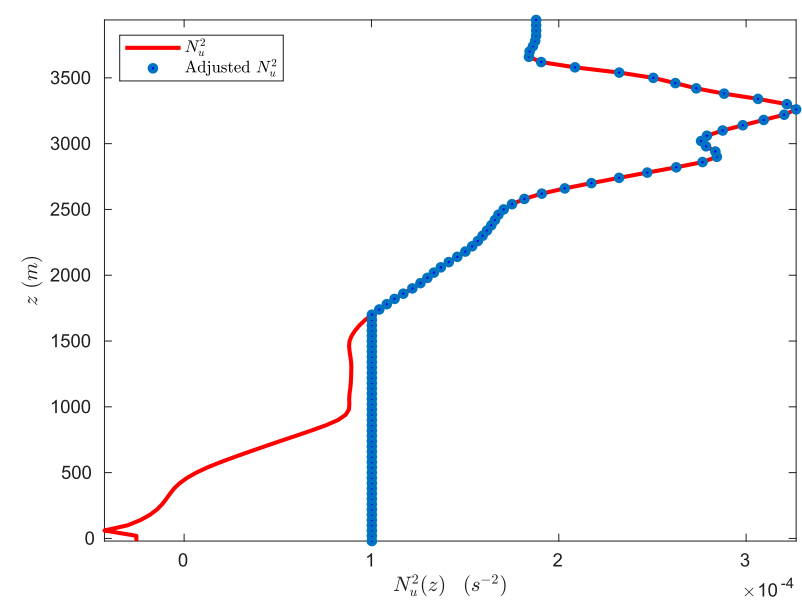

FIG. 3. An illustration of the adjustment process for $N_{u}^{2}$. The red curve shows $N_{u}^{2}$ and the blue shows how it is changed for use in the EOF analysis.

are centered to have a horizontal mean of zero at each vertical level. Finally, and perhaps most importantly, $N_{u}^{2}(z)$ and $N_{s}^{2}(z)$ are not uniformly positive over the domain. Note that while the buoyancy frequencies depend on both space and time, we approximate them as purely height-dependent functions according to (11a) and (11b). As can be seen in Fig. 3, $N_{u}^{2}(z)<0$ in the lower $500 \mathrm{~m}$ of the domain, within the subcloud layer, which is well mixed. To use $N_{u}^{2}(z)$ to define a positive energy, it will need to be modified in this layer, and we modify it by simply increasing $N_{u}^{2}(z)$ to be $10^{-4} \mathrm{~s}^{-1}$ at any level where it is originally less than $10^{-4} \mathrm{~s}^{-1}$, as illustrated in Fig. 3 . This type of modification is also tacitly in place in other studies whenever a standard $L^{2}$ energy is used to analyze data within a well-mixed or unstable layer. For the other buoyancy frequency $N_{s}^{2}(z)$, negative values occur over a wider range of heights, so we simply set $N_{s}^{2}(z)=(1 / 10) N_{u}^{2}(z)$ at all heights to define a positive $N_{s}^{2}(z)$. Other choices of scale factors larger than $1 / 10$, such as $1 / 2$, were also considered, but they were found to produce leading EOFs that were similar to the case of an energy without phase changes; it would be interesting to examine this choice in further detail in the future. Overall, these definitions of $N_{u}^{2}(z)$ and $N_{s}^{2}(z)$ involve some modification from the theoretical piecewise-quadratic energy definition, but they preserve the essence of piecewise-quadratic energy in the sense that $N_{s}^{2}(z)$ will be significantly smaller than $N_{u}^{2}(z)$ and will therefore create a naturally enhanced weighting of buoyancy fluctuations in the saturated phase (i.e., within clouds), since the reciprocal $N_{s}^{-2}(z)$ is the weighting factor.

The comparison of EOFs for the standard $L^{2}$ energy and the piecewise-quadratic energy are shown in Figs. 4-6. In terms of variance or energy, in Fig. 4, the
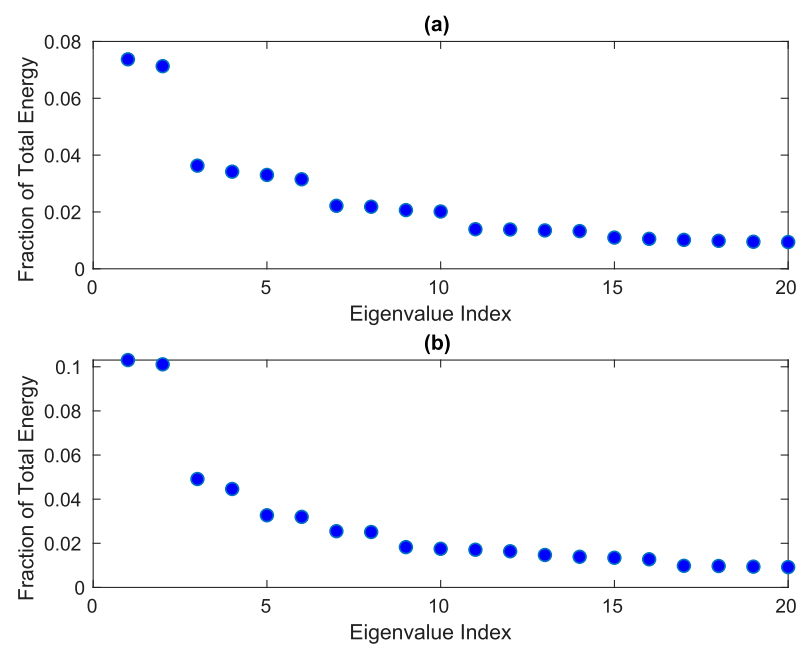

FIG. 4. Eigen values of (a) the $\mathbf{B}$ covariance matrix as a fraction of total energy and (b) the $b_{u}$ covariance matrix as a fraction of total energy.

two cases appear to capture roughly similar amounts of energy in each mode. However, in terms of EOF structures, the two cases show substantial differences. Figures 5 and 6 show the first four EOFs of $b_{u}$ and $B$, respectively. The most important feature is that the first two $b_{u}$ modes are limited to the lower part of the domain, whereas the first two $B$ modes extend into the upper part of the domain, which means that the $B$ modes capture cloud variability while the $b_{u}$ modes capture mainly the subcloud layer.

One way of understanding the significance of these qualitative differences of the modes is to look at time series of the fraction of the domain covered by clouds. As can be seen in Figs. 1 and 2, the domain is covered by few clouds for most of the simulation. It is, however, punctuated by brief periods of relatively high cloud coverage, which suggests that clouds display intermittency in both space and time. In terms of the standard $L^{2}$ energy, the cloud variability contributes little to the energy, due to its intermittency. On the other hand, the piecewise-quadratic energy gives different weight, $N_{u}^{-2}$ versus $N_{s}^{-2}$, to the unsaturated versus saturated regions, which allows the cloud variability to make a substantial contribution to the energy and manifest itself in the leading EOFs.

\section{Discussion and conclusions}

In summary, we have examined energetics for moist atmospheres with phase changes. We first considered a Boussinesq system with moisture and phase changes, and in one of the main results, we found a piecewise-quadratic, positive definite conserved energy. For this same system, we also considered a second definition of an energy, based on a potential energy $\Pi$ that generalizes a $\rho g z$-like 

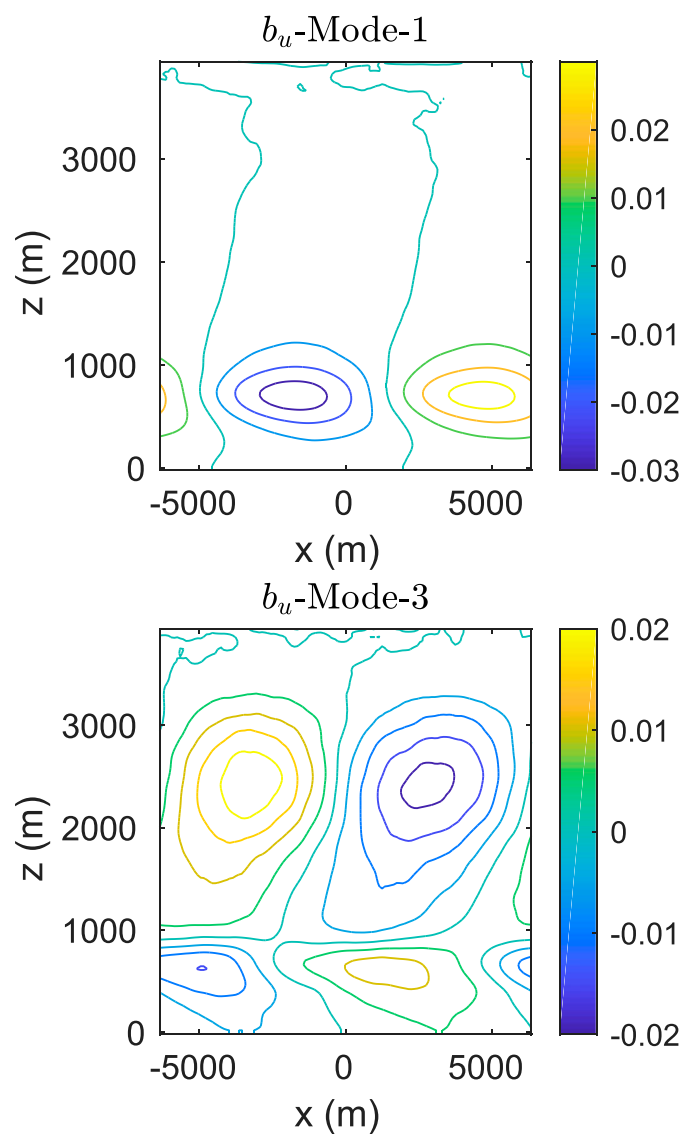
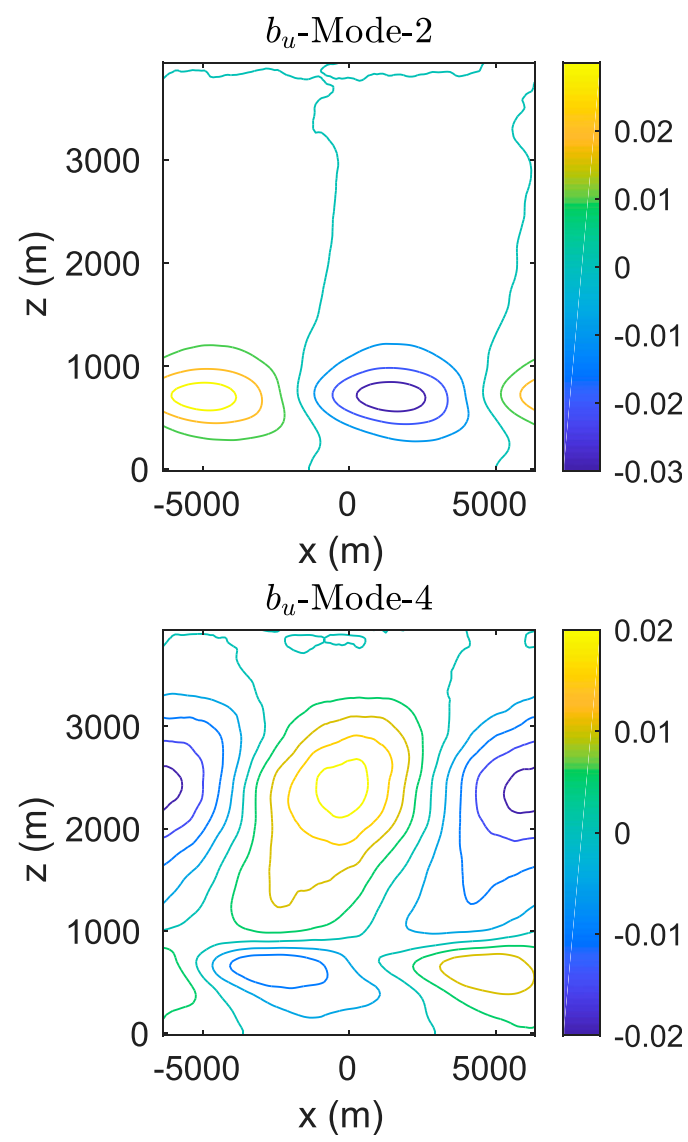

FIG. 5. The first four EOFs based on the $b_{u}$ covariance with standard $L^{2}$ energy norm.

potential energy. While the П-based energy is not manifestly positive definite, it was shown to be equal to the piecewise-quadratic energy, plus some additional material invariant terms.

Two aspects are perhaps most important in generalizing the dry quadratic energy to the moist piecewisequadratic energy. First, by using Heaviside functions $H_{u}$ and $H_{s}$ to represent phase changes, it becomes relatively straightforward to account for the distinction between the two phases. For example, the Heaviside functions are useful in carrying out the integration in the П-based potential energy, which then demonstrates the relationship between the П-based energy and the piecewisequadratic energy. Second, the contribution of the variable $M$ is related to an additional eigenmode of the moist system that is not present in the dry system, and it is natural that one component of the moist energy would be associated with this additional eigenmode.

Increasing amounts of complexity were then incorporated beyond the moist Boussinesq equations. As a first step, in section 3, the moist anelastic equations were considered. For the anelastic system, the energy is not quadratic, even in the dry case. Nevertheless, it was shown that the potential energy can be decomposed into three components: buoyant potential energy in the unsaturated phase, buoyant potential energy in the saturated phase, and a moist latent energy that is analogous to the $M$ contribution from the Boussinesq case. In adding further complexity, we considered Kessler warm-rain microphysics, in which case we cannot in general obtain a conserved energy due to the presence of source terms representing rain and cloud processes. As a final case, we considered FARE microphysics, which is a simplified version of warm-rain microphysics. The case of FARE microphysics is interesting because, like Kessler microphysics, it includes precipitation; however, unlike Kessler microphysics, FARE microphysics was shown to have a piecewise-quadratic energy, and the energy is conserved, aside from a signdefinite dissipation term due to precipitation at the surface.

As an application, we considered an empirical orthogonal analysis that uses a piecewise-quadratic energy as weighted norm, in place of the standard $L^{2}$ norm. In comparing the leading EOFs produced by these two cases (i.e., by using piecewise-quadratic norm versus standard $L^{2}$ norm), substantially different EOF modes 

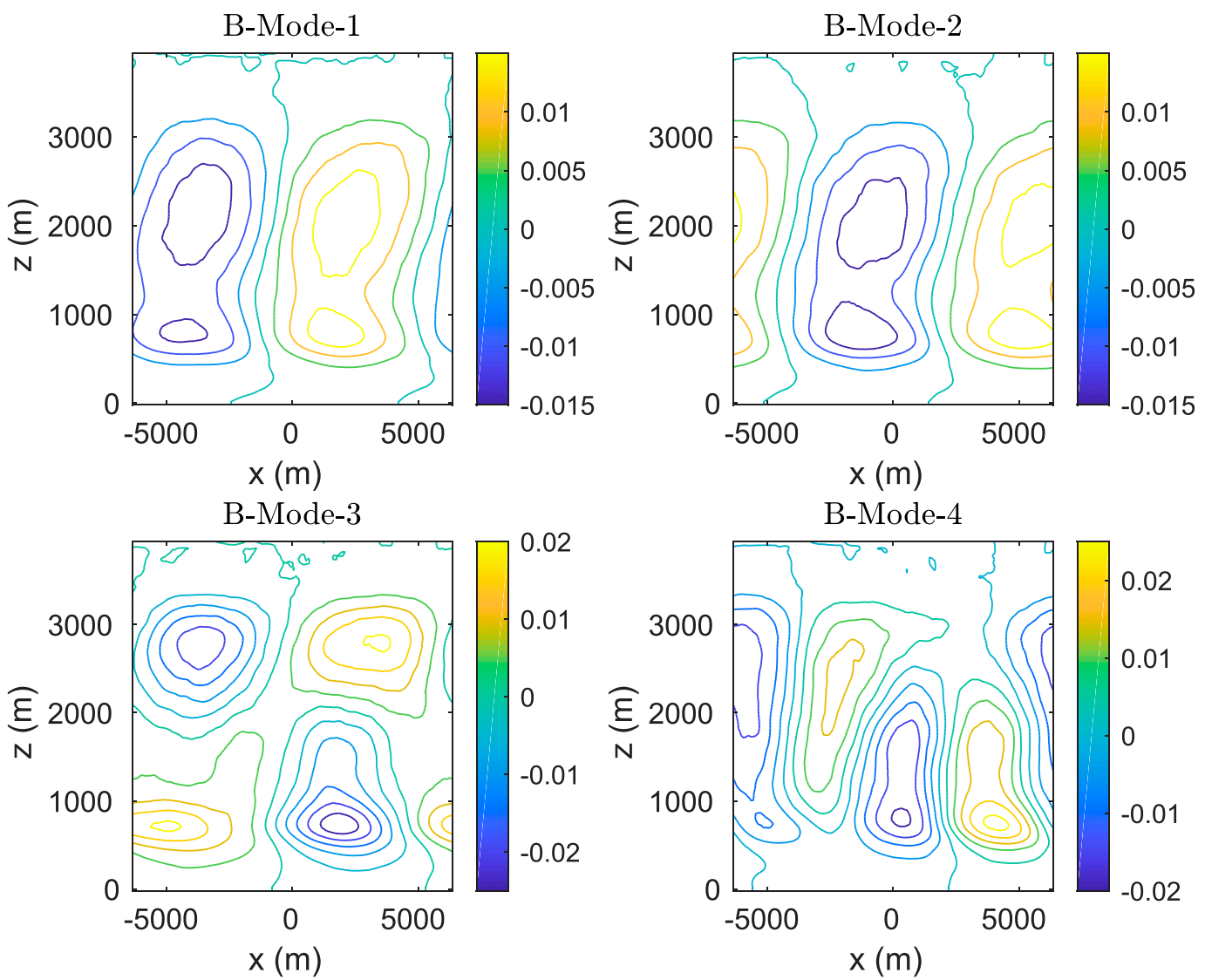

FIG. 6. The first four EOFs based on the $B$ covariance with phase changes in the energy.

were seen. The piecewise-quadratic norm is a weighted norm that incorporates information about the phase changes, and, as such, it produces EOFs that are representative of cloud variability. It would be interesting in the future to further investigate and refine this type of weighted norm and its applicability to observational and computational datasets.

Acknowledgments. The authors thank Rupert Klein and two anonymous reviewers for helpful comments. This research is partially supported by NSF Grants AGS-1443325 and DMS-1907667 and by the University of Wisconsin-Madison Office of the Vice Chancellor for Research and Graduate Education with funding from the Wisconsin Alumni Research Foundation.

\section{APPENDIX A}

Reformulation of Moist Boussinesq Equations in Terms of Unsaturated and Saturated Buoyancies and Extension to the Kessler Scheme

We derive (8a)-(8d). Our starting point is the system

$$
\begin{aligned}
\frac{D \mathbf{u}}{D t} & =-\nabla \phi+b \hat{\mathbf{z}}, \\
\frac{D \theta_{e}}{D t}+\frac{d \tilde{\theta}_{e}}{d z} w & =0, \\
\frac{D q_{t}}{D t}+\frac{d \tilde{q}_{t}}{d z} w & =0, \\
\nabla \cdot \mathbf{u} & =0
\end{aligned}
$$

where $p^{\prime}$ is the pressure, $\rho_{0}$ is a constant background density, $\phi=p^{\prime} / \rho_{0}, \mathbf{u}(\mathbf{x}, t)$ is the velocity vector, $\theta_{e}$ is the equivalent potential temperature anomaly, and $q_{t}$ is the anomalous total water mixing ratio. The buoyancy, $b$, can be written in terms of $\theta_{e}, q_{t}$, and $z$, and will be described below. We assume that all thermodynamic variables have been decomposed into background functions of height and anomalous parts, so that, for example, $\theta_{e}^{\text {tot }}=\tilde{\theta}_{e}(z)+\theta_{e}(\mathbf{x}, t)$.

The total water $q_{t}$ is the sum of water vapor $q_{v}$ and liquid water $q_{l}$ and can be written as

$$
q_{t}=q_{v}+q_{l}
$$


To obtain equations for $q_{v}$ and $q_{l}$, we adopt a prescribed saturation mixing ratio $q_{v s}^{\text {tot }}(z)$ that depends on height alone, in part because it allows explicit expressions to be derived in what follows. In making this approximation, we have assumed that $T^{\mathrm{tot}}$ and $p^{\mathrm{tot}}$ are close to the background states $\tilde{p}$ and $\tilde{T}$, so that $q_{v s}^{\text {tot }}\left(T^{\text {tot }}, p^{\text {tot }}\right) \approx q_{v s}^{\text {tot }}[\tilde{T}(z), \tilde{p}(z)]$. We then have

$$
\begin{aligned}
& q_{v}=\min \left[q_{t}, q_{v s}(z)\right], \\
& q_{l}=\max \left[0, q_{t}-q_{v s}(z)\right] .
\end{aligned}
$$

The equivalent potential temperature is defined as

$$
\theta_{e}=\theta+\frac{L_{v}}{c_{p}} q_{v}
$$

where $\theta$ is the potential temperature, the latent heat factor is $L_{v} \approx 2.5 \times 10^{6} \mathrm{~J} \mathrm{~kg}^{-1}$, and specific heat is $c_{p} \approx$ $10^{3} \mathrm{~J} \mathrm{~kg}^{-1} \mathrm{~K}^{-1}$; this definition of $\theta_{e}$ is a linearization of usual definitions (e.g., Emanuel 1994; Stevens 2005), and this linearized version is helpful for the exposition here. Using the equations above, we can obtain an expression for the potential temperature:

$$
\theta=\left\{\begin{array}{ccc}
\theta_{e}-\frac{L_{v}}{c_{p}} q_{t} & \text { if } & q_{t}<q_{v s}(z) \\
\theta_{e}-\frac{L_{v}}{c_{p}} q_{v s}(z) & \text { if } & q_{t} \geq q_{v s}(z) .
\end{array}\right.
$$

The buoyancy $b$ is defined as

$$
b=g\left(\frac{\theta}{\theta_{0}}+R_{v d} q_{v}-q_{l}\right),
$$

where $\theta_{0} \approx 300 \mathrm{~K}$ is a constant background potential temperature, $g \approx 9.8 \mathrm{~m} \mathrm{~s}^{-2}$ is the acceleration due to gravity and $R_{v d}=\left(R_{v} / R_{d}\right)-1 \approx 0.61$, where $R_{d}$ is the gas constant for dry air and $R_{v}$ is the gas constant for water vapor. The buoyancy changes form based on phase, so that we can write

$$
b=b_{u} H_{u}+b_{s} H_{s},
$$

where $b_{u}$ and $b_{s}$ are defined to be the buoyancies in unsaturated and saturated regions, respectively. The functions $H_{u}$ and $H_{s}$ are Heaviside functions that indicate unsaturated and saturated phases, respectively, and are defined as

$$
H_{u}=\left\{\begin{array}{lll}
1 & \text { if } & q_{t}<q_{v s}(z) \\
0 & \text { if } & q_{t} \geq q_{v s}(z)
\end{array}\right.
$$

and $H_{s}=1-H_{u}$. Using the equations above, we can show that $b_{u}$ and $b_{s}$ are defined as

$$
\begin{aligned}
& b_{u}=g\left[\frac{\theta_{e}}{\theta_{0}}+\left(R_{v d}-\frac{L_{v}}{c_{p} \theta_{0}}\right) q_{t}\right], \\
& b_{s}=g\left[\frac{\theta_{e}}{\theta_{0}}+\left(R_{v d}-\frac{L_{v}}{c_{p} \theta_{0}}+1\right) q_{v s}(z)-q_{t}\right],
\end{aligned}
$$

Differentiating (A9a) and (A9b), and using (A1b) and (A1c) results in

$$
\begin{aligned}
& \frac{D b_{u}}{D t}+N_{u}^{2} w=0, \\
& \frac{D b_{s}}{D t}+N_{s}^{2} w=0,
\end{aligned}
$$

where the buoyancy frequencies $N_{u}^{2}$ and $N_{s}^{2}$ are

$$
\begin{aligned}
& N_{u}^{2}=g \frac{d}{d z}\left[\frac{\tilde{\theta}_{e}}{\theta_{0}}+\left(R_{v d}-\frac{L_{v}}{c_{p} \theta_{0}}\right) \tilde{q}_{t}\right], \\
& N_{s}^{2}=g \frac{d}{d z}\left[\frac{\tilde{\theta}_{e}}{\theta_{0}}-\left(R_{v d}-\frac{L_{v}}{c_{p} \theta_{0}}+1\right) q_{v s}(z)-\tilde{q}_{t}\right] .
\end{aligned}
$$

Note that an advantage of formulating the equations in terms of $b_{u}$ and $b_{s}$ is that it leads to a particularly simple phase interface condition; $b_{u}=b_{s}$. This can be seen by observing that when $q_{t}=q_{v s}$, (A9a) and (A9b) are equal.

In the Kessler scheme, the diagnostic equations for water vapor $q_{v}$ and cloud water $q_{c}$ are

$$
q_{v}=\left\{\begin{array}{ccc}
q_{t}-q_{r} & \text { if } & q_{t}-q_{r} \leq q_{v s}(z) \\
q_{v s}(z) & \text { if } & q_{t}-q_{r}>q_{v s}(z)
\end{array}\right.
$$

and

$$
q_{c}=q_{t}-q_{r}-q_{v} .
$$

The buoyancy now includes additional moisture terms and takes the form

$$
b=g\left(\frac{\theta}{\theta_{0}}+R_{v d} q_{v}-q_{r}-q_{c}\right) .
$$

In saturated regions, $q_{t}-q_{r} \geq q_{v s}$, and in unsaturated regions, $q_{t}-q_{r}<q_{v s}$, and it follows that the buoyancy in the unsaturated and saturated regions, are, respectively,

$$
b_{u}=g\left[\frac{\theta_{e}}{\theta_{0}}+\left(R_{v d}-\frac{L_{v}}{c_{p} \theta_{0}}\right) q_{t}-\left(R_{v d}-\frac{L_{v}}{c_{p} \theta_{0}}+1\right) q_{r}\right]
$$

and 


$$
b_{s}=g\left[\frac{\theta_{e}}{\theta_{0}}+\left(R_{v d}-\frac{L_{v}}{c_{p} \theta_{0}}+1\right) q_{v s}-q_{t}\right],
$$

where the saturation condition in terms of $b_{u}$ and $b_{s}$ is still conveniently expressed as $b_{u}=b_{s}$.

\section{APPENDIX B}

\section{Integrating II for the Boussinesq Equations}

In this section, we provide the details of how to perform the integration of the potential energy $\Pi$ in sections $2 \mathrm{~d}$ and 2e. First, we integrate the term $-\left(b_{u}^{\text {tot }}-N_{u}^{2} z^{\prime}\right) H_{u}$. After an integration by parts, noting that, formally,

$$
\begin{aligned}
& \frac{d}{d z} H\left[b_{u}^{\mathrm{tot}}-b_{s}^{\mathrm{tot}}-\left(N_{u}^{2}-N_{s}^{2}\right) z\right] \\
& \quad=-\left(N_{u}^{2}-N_{s}^{2}\right) \delta\left[b_{u}^{\mathrm{tot}}-b_{s}^{\mathrm{tot}}-\left(N_{u}^{2}-N_{s}^{2}\right) z\right],
\end{aligned}
$$

we have

$$
\begin{aligned}
-\int_{a}^{z}\left(b_{u}^{\mathrm{tot}}-N_{u}^{2} z^{\prime}\right) H_{u} d z^{\prime}= & \left.\frac{\left(b_{u}^{\mathrm{tot}}-N_{u}^{2} z^{\prime}\right)^{2}}{2 N_{u}^{2}} H\left[b_{u}^{\mathrm{tot}}-b_{s}^{\mathrm{tot}}-\left(N_{u}^{2}-N_{s}^{2}\right) z^{\prime}\right]\right|_{a} ^{z} \\
& +\int_{a}^{z} \frac{\left(b_{u}^{\mathrm{tot}}-N_{u}^{2} z^{\prime}\right)^{2}}{2 N_{u}^{2}}\left(N_{u}^{2}-N_{s}^{2}\right) \delta\left[b_{u}^{\mathrm{tot}}-b_{s}^{\mathrm{tot}}-\left(N_{u}^{2}-N_{s}^{2}\right) z^{\prime}\right] d z^{\prime} .
\end{aligned}
$$

The first term on the right hand side of (B1) is straightforward to evaluate:

$$
\begin{aligned}
\left.\frac{\left(b_{u}^{\mathrm{tot}}-N_{u}^{2} z^{\prime}\right)^{2}}{2 N_{u}^{2}} H\left[b_{u}^{\mathrm{tot}}-b_{s}^{\mathrm{tot}}-\left(N_{u}^{2}-N_{s}^{2}\right) z^{\prime}\right]\right|_{a} ^{z} & =\frac{\left(b_{u}^{\mathrm{tot}}-N_{u}^{2} z\right)^{2}}{2 N_{u}^{2}} H\left[b_{u}^{\mathrm{tot}}-b_{s}^{\mathrm{tot}}-\left(N_{u}^{2}-N_{s}^{2}\right) z\right] \\
& -\frac{\left(b_{u}^{\mathrm{tot}}-N_{u}^{2} a\right)^{2}}{2 N_{u}^{2}} H\left[b_{u}^{\mathrm{tot}}-b_{s}^{\mathrm{tot}}-\left(N_{u}^{2}-N_{s}^{2}\right) a\right] \\
& =\frac{b_{u}^{2}}{2 N_{u}^{2}} H_{u}-\frac{\left[b_{u}+N_{u}^{2}(z-a)\right]^{2}}{2 N_{u}^{2}} H\left\{b_{u}-b_{s}+\left[N_{u}^{2}-N_{s}^{2}(z-a)\right]\right\},
\end{aligned}
$$

where we have used the fact that $b_{u}^{\text {tot }}=b_{u}-N_{u}^{2} z$ and $H_{u}=H\left(b_{u}-b_{s}\right)$.

Now we focus on the second term on the right hand side of (B1). To integrate this term, we multiply the integrand by the characteristic function of the interval $[a, z]$, which is $H\left(z^{\prime}-a\right)-H\left(z^{\prime}-z\right)$, and integrate over the entire real line. Doing this ensures that the zero of the delta function's argument, $z^{\prime}=\left(b_{u}^{\text {tot }}-b_{s}^{\text {tot }}\right) /\left(N_{u}^{2}-N_{s}^{2}\right)$, is actually within the region of integration. Therefore,

$$
\begin{aligned}
& \int_{a}^{z} \frac{\left(b_{u}^{\mathrm{tot}}-N_{u}^{2} z^{\prime}\right)^{2}}{2 N_{u}^{2}}\left(N_{u}^{2}-N_{s}^{2}\right) \delta\left[b_{u}^{\mathrm{tot}}-b_{s}^{\mathrm{tot}}-\left(N_{u}^{2}-N_{s}^{2}\right) z^{\prime}\right] d z^{\prime} \\
& \quad=\int_{-\infty}^{\infty}\left[H\left(z^{\prime}-a\right)-H\left(z^{\prime}-z\right)\right] \frac{\left(b_{u}^{\mathrm{tot}}-N_{u}^{2} z^{\prime}\right)^{2}}{2 N_{u}^{2}}\left(N_{u}^{2}-N_{s}^{2}\right) \delta\left[b_{u}^{\mathrm{tot}}-b_{s}^{\mathrm{tot}}-\left(N_{u}^{2}-N_{s}^{2}\right) z^{\prime}\right] d z^{\prime} \\
& \quad=\left[H\left(\frac{b_{u}^{\mathrm{tot}}-b_{s}^{\mathrm{tot}}}{N_{u}^{2}-N_{s}^{2}}-a\right)-H\left(\frac{b_{u}^{\mathrm{tot}}-b_{s}^{\mathrm{tot}}}{N_{u}^{2}-N_{s}^{2}}-z\right)\right] \frac{1}{2 N_{u}^{2}}\left[b_{u}^{\mathrm{tot}}-N_{u}^{2}\left(\frac{b_{u}^{\mathrm{tot}}-b_{s}^{\mathrm{tot}}}{N_{u}^{2}-N_{s}^{2}}\right)\right]^{2} \\
& \quad=\left\{H\left[b_{u}^{\mathrm{tot}}-b_{s}^{\mathrm{tot}}-\left(N_{u}^{2}-N_{s}^{2}\right) a\right]-H\left[b_{u}^{\mathrm{tot}}-b_{s}^{\mathrm{tot}}-\left(N_{u}^{2}-N_{s}^{2}\right) z\right]\right\} \frac{1}{2 N_{u}^{2}}\left[b_{u}^{\mathrm{tot}}-N_{u}^{2}\left(\frac{b_{u}^{\mathrm{tot}}-b_{s}^{\mathrm{tot}}}{N_{u}^{2}-N_{s}^{2}}\right)\right]^{2},
\end{aligned}
$$


where we have used the fact that

$$
H\left(\frac{b_{u}^{\mathrm{tot}}-b_{s}^{\mathrm{tot}}}{N_{u}^{2}-N_{s}^{2}}-a\right)=H\left[b_{u}^{\mathrm{tot}}-b_{s}^{\mathrm{tot}}-\left(N_{u}^{2}-N_{s}^{2}\right) a\right]
$$

$$
H\left(\frac{b_{u}^{\mathrm{tot}}-b_{s}^{\mathrm{tot}}}{N_{u}^{2}-N_{s}^{2}}-z\right)=H\left[b_{u}^{\mathrm{tot}}-b_{s}^{\mathrm{tot}}-\left(N_{u}^{2}-N_{s}^{2}\right) z\right] .
$$

Therefore,

and

$$
\begin{aligned}
-\int_{a}^{z}\left(b_{u}^{\mathrm{tot}}-N_{u}^{2} z^{\prime}\right) H_{u} d z^{\prime}= & \frac{b_{u}^{2}}{2 N_{u}^{2}} H_{u}-\frac{\left[b_{u}+N_{u}^{2}(z-a)\right]^{2}}{2 N_{u}^{2}} H\left\{b_{u}-b_{s}+\left[N_{u}^{2}-N_{s}^{2}(z-a)\right]\right\} \\
& -\frac{1}{2 N_{u}^{2}}\left[b_{u}^{\mathrm{tot}}-N_{u}^{2}\left(\frac{b_{u}^{\mathrm{tot}}-b_{s}^{\mathrm{tot}}}{N_{u}^{2}-N_{s}^{2}}\right)\right]^{2} H\left[b_{u}^{\mathrm{tot}}-b_{s}^{\mathrm{tot}}-\left(N_{u}^{2}-N_{s}^{2}\right) z\right] \\
& +\frac{1}{2 N_{u}^{2}}\left[b_{u}^{\mathrm{tot}}-N_{u}^{2}\left(\frac{b_{u}^{\mathrm{tot}}-b_{s}^{\mathrm{tot}}}{N_{u}^{2}-N_{s}^{2}}\right)\right]^{2} H\left[b_{u}^{\mathrm{tot}}-b_{s}^{\mathrm{tot}}-\left(N_{u}^{2}-N_{s}^{2}\right) a\right],
\end{aligned}
$$

which provides an explicit expression for the integral in (B1). of $-\left(b_{s}^{\text {tot }}-N_{s}^{2} z\right) H_{s}$. To evaluate this latter integral,

Similar to the integral of $\left(b_{u}^{\text {tot }}-N_{u}^{2} z^{\prime}\right) H_{u}$ from (B1), a procedure analogous to the one just carried shows the potential energy $\Pi$ also includes an integral that

$$
\begin{aligned}
-\int_{a}^{z}\left(b_{s}^{\mathrm{tot}}-N_{s}^{2} z^{\prime}\right) H_{s} d z^{\prime}= & \frac{b_{s}^{2}}{2 N_{s}^{2}} H_{s}-\frac{1}{2 N_{s}^{2}}\left[b_{s}+N_{s}^{2}(z-a)\right]^{2} \\
& +\frac{\left[b_{s}+N_{s}^{2}(z-a)\right]^{2}}{2 N_{s}^{2}} H\left\{b_{u}-b_{s}+\left[N_{u}^{2}-N_{s}^{2}(z-a)\right]\right\} \\
& +\frac{1}{2 N_{s}^{2}}\left[b_{s}^{\mathrm{tot}}-N_{s}^{2}\left(\frac{b_{u}^{\mathrm{tot}}-b_{s}^{\mathrm{tot}}}{N_{u}^{2}-N_{s}^{2}}\right)\right]^{2} H\left[b_{u}^{\mathrm{tot}}-b_{s}^{\mathrm{tot}}-\left(N_{u}^{2}-N_{s}^{2}\right) z\right] \\
& -\frac{1}{2 N_{s}^{2}}\left[b_{s}^{\mathrm{tot}}-N_{s}^{2}\left(\frac{b_{u}^{\mathrm{tot}}-b_{s}^{\mathrm{tot}}}{N_{u}^{2}-N_{s}^{2}}\right)\right]^{2} H\left[b_{u}^{\mathrm{tot}}-b_{s}^{\mathrm{tot}}-\left(N_{u}^{2}-N_{s}^{2}\right) a\right]
\end{aligned}
$$

Adding (B4) and (B5) and a great deal of simplifying yields

$$
\begin{aligned}
\Pi= & \frac{b_{u}^{2}}{2 N_{u}^{2}} H_{u}+\frac{b_{s}^{2}}{2 N_{s}^{2}} H_{s}+\frac{N_{u}^{2} N_{s}^{2}}{2\left(N_{u}^{2}-N_{s}^{2}\right)} M^{2} H_{u} \\
& -\frac{1}{2 N_{s}^{2}}\left[b_{s}+N_{s}^{2}(z-a)\right]^{2} \\
& -\frac{1}{2 N_{u}^{2}}\left[b_{u}+N_{u}^{2}(z-a)\right]^{2} H\left[b_{u}-b_{s}+\left(N_{u}^{2}-N_{s}^{2}\right)(z-a)\right] \\
& +\frac{1}{2 N_{s}^{2}}\left[b_{s}+N_{s}^{2}(z-a)\right]^{2} H\left[b_{u}-b_{s}+\left(N_{u}^{2}-N_{s}^{2}\right)(z-a)\right] \\
& -\frac{N_{u}^{2} N_{s}^{2}}{2\left(N_{u}^{2}-N_{s}^{2}\right)} M^{2} H\left[b_{u}-b_{s}+\left(N_{u}^{2}-N_{s}^{2}\right)(z-a)\right] .
\end{aligned}
$$

Notice that, on the right-hand side, the first three terms are components of the piecewise quadratic energy, and the rest of the terms are functions of three material invariants: $M, b_{u}^{\text {tot }}=b_{u}+N_{u}^{2} z$, and $b_{s}^{\text {tot }}=b_{s}+N_{s}^{2} z$. To conclude this 
appendix, we point out that choosing $a=z_{r}$ would result in $E_{1}=E_{2}$, where $E_{1}$ is given by (21) and $E_{2}$ by (26).

\section{REFERENCES}

Andrews, D. G., 1981: A note on potential energy density in a stratified compressible fluid. J. Fluid Mech., 107, 227-236, https://doi.org/10.1017/S0022112081001754.

Bretherton, C. S., 1987: A theory for nonprecipitating moist convection between two parallel plates. Part I: Thermodynamics and "linear" solutions. J. Atmos. Sci., 44, 1809-1827, https://doi.org/ 10.1175/1520-0469(1987)044<1809:ATFNMC > 2.0.CO;2.

Chen, S., and S. N. Stechmann, 2016: Nonlinear traveling waves for the skeleton of the Madden-Julian oscillation. Commun. Math. Sci., 14, 571-592, https://doi.org/10.4310/CMS.2016.v14.n2.a11.

Cuijpers, J. W. M., and P. G. Duynkerke, 1993: Large eddy simulation of trade wind cumulus clouds. J. Atmos. Sci., 50, 3894-3908, https:// doi.org/10.1175/1520-0469(1993)050<3894:LESOTW>2.0.CO;2.

Emanuel, K. A., 1994: Atmospheric Convection. Oxford University Press, $592 \mathrm{pp}$

Frierson, D. M. W., A. J. Majda, and O. M. Pauluis, 2004: Large scale dynamics of precipitation fronts in the tropical atmosphere: a novel relaxation limit. Commun. Math. Sci., 2, 591626, https://doi.org/10.4310/CMS.2004.v2.n4.a3.

Grabowski, W. W., and T. L. Clark, 1993: Cloud-environment interface instability. Part II: Extension to three spatial dimensions. J. Atmos. Sci., 50, 555-573, https://doi.org/10.1175/ 1520-0469(1993)050<0555:CEIIPI >2.0.CO;2.

__ , and P. K. Smolarkiewicz, 1996: Two-time-level semiLagrangian modeling of precipitating clouds. Mon. Wea. Rev., 124, 487-497, https://doi.org/10.1175/1520-0493(1996) $124<0487$ :TTLSLM $>2.0$. CO 2 .

Hernandez-Duenas, G., A. J. Majda, L. M. Smith, and S. N. Stechmann, 2013: Minimal models for precipitating turbulent convection. J. Fluid Mech., 717, 576-611, https://doi.org/ 10.1017/jfm.2012.597.

_ L. M. Smith, and S. N. Stechmann, 2015: Stability and instability criteria for idealized precipitating hydrodynamics. J. Atmos. Sci., 72, 2379-2393, https://doi.org/10.1175/JAS-D-14-0317.1.

,-- , and 2019: Weak- and strong-friction limits of parcel models: Comparisons and stochastic convective initiation time. Quart. J. Roy. Meteor. Soc., 145, 2272-2291, https:// doi.org/10.1002/qj.3557.

Holliday, D., and M. E. McIntyre, 1981: On potential energy density in an incompressible, stratified fluid. J. Fluid Mech. 107, 221-225, https://doi.org/10.1017/S0022112081001742.

Ingersoll, A. P., 2005: Boussinesq and anelastic approximations revisited: Potential energy release during thermobaric instability. J. Phys. Oceanogr., 35, 1359-1369, https://doi.org/ 10.1175/JPO2756.1.

Kessler, E., 1969: On the Distribution and Continuity of Water Substance in Atmospheric Circulations. Meteor. Monogr., No. 32, Amer. Meteor. Soc., 84 pp.

Kuo, H. L., 1961: Convection in conditionally unstable atmosphere. Tellus, 13, 441-459, https://doi.org/10.3402/tellusa.v13i4.9516.

Lorenz, E. N., 1955: Available potential energy and the maintenance of the general circulation. Tellus, 7, 157-167, https:// doi.org/10.3402/tellusa.v7i2.8796.

_ 1978: Available energy and the maintenance of a moist circulation. Tellus, 30, 15-31, https://doi.org/10.3402/tellusa.v30i1.10308.

Moncrieff, M. W., and M. J. Miller, 1976: The dynamics and simulation of tropical cumulonimbus and squall lines. Quart.
J. Roy. Meteor. Soc., 102, 373-394, https://doi.org/10.1002/ qj. 49710243208 .

Pauluis, O., 2007: Sources and sinks of available potential energy in a moist atmosphere. J. Atmos. Sci., 64, 2627-2641, https:// doi.org/10.1175/JAS3937.1.

— 2008: Thermodynamic consistency of the anelastic approximation for a moist atmosphere. J. Atmos. Sci., 65, 2719-2729, https://doi.org/10.1175/2007JAS2475.1.

_ , and J. Schumacher, 2010: Idealized moist Rayleigh-Bénard convection with piecewise linear equation of state. Commun. Math. Sci., 8, 295-319, https://doi.org/10.4310/CMS.2010.v8.n1.a15. , and — 2011: Self-aggregation of clouds in conditionally unstable moist convection. Proc. Natl. Acad. Sci. USA, 108, 12 623-12 628, https://doi.org/10.1073/pnas.1102339108.

Rauber, R. M., and Coauthors, 2007: Rain in shallow cumulus over the ocean: The RICO campaign. Bull. Amer. Meteor. Soc., 88, 1912-1928, https://doi.org/10.1175/BAMS-88-12-1912.

Seifert, A., and K. D. Beheng, 2001: A double-moment parameterization for simulating autoconversion, accretion and selfcollection. Atmos. Res., 59-60, 265-281, https://doi.org/10.1016/ S0169-8095(01)00126-0.

— rameterization for mixed-phase clouds. Part 1: Model description. Meteor. Atmos. Phys., 92, 45-66, https://doi.org/ 10.1007/s00703-005-0112-4.

Smith, L. M., and S. N. Stechmann, 2017: Precipitating quasigeostrophic equations and potential vorticity inversion with phase changes. J. Atmos. Sci., 74, 3285-3303, https://doi.org/10.1175/ JAS-D-17-0023.1.

Stansifer, E. M., P. A. O'Gorman, and J. I. Holt, 2017: Accurate computation of moist available potential energy with the Munkres algorithm. Quart. J. Roy. Meteor. Soc., 143, 288-292, https://doi.org/10.1002/qj.2921.

Stechmann, S. N., 2014: Multiscale eddy simulation for moist atmospheric convection: Preliminary investigation. J. Comput. Phys., 271, 99-117, https://doi.org/10.1016/j.jcp.2014.02.009.

— and A. J. Majda, 2006: The structure of precipitation fronts for finite relaxation time. Theor. Comput. Fluid Dyn., 20, 377404, https://doi.org/10.1007/s00162-006-0014-1.

Stevens, B., 2005: Atmospheric moist convection. Annu. Rev. Earth Planet. Sci., 33, 605-643, https://doi.org/10.1146/ annurev.earth.33.092203.122658.

2007: On the growth of layers of nonprecipitating cumulus convection. J. Atmos. Sci., 64, 2916-2931, https://doi.org/ 10.1175/JAS3983.1.

_- and Coauthors, 2005: Evaluation of large-eddy simulations via observations of nocturnal marine stratocumulus. Mon. Wea. Rev., 133, 1443-1462, https://doi.org/10.1175/MWR2930.1.

Tailleux, R., 2013: Available potential energy density for a multicomponent Boussinesq fluid with arbitrary nonlinear equation of state. J. Fluid Mech., 735, 499-518, https://doi.org/10.1017/jfm.2013.509.

Vallis, G., 2006: Atmospheric and Oceanic Fluid Dynamics: Fundamentals and Large-scale Circulation. Cambridge University Press, $745 \mathrm{pp}$.

Vallis, G. K., D. J. Parker, and S. M. Tobias, 2019: A simple system for moist convection: The Rainy-Bénard model. J. Fluid Mech., 862, 162-199, https://doi.org/10.1017/jfm.2018.954.

Wetzel, A. N., L. M. Smith, S. N. Stechmann, and J. E. Martin, 2019: Balanced and unbalanced components of moist atmospheric flows with phase changes. Chin. Ann. Math. B, in press.

Young, W. R., 2010: Dynamic enthalpy, conservative temperature, and the seawater Boussinesq approximation. J. Phys. Oceanogr., 40, 394-400, https://doi.org/10.1175/2009JPO4294.1. 